\title{
Inflación y rendimientos en mercados emergentes: el caso de Argentina
}

\author{
PEsce, GABRIELA \\ Universidad Nacional del Sur (Argentina) \\ Correo electrónico: gabriela.pesce@uns.edu.ar \\ PEDRoni, Florencia Verónica \\ Consejo Nacional de Investigaciones Científicas y Técnicas, Universidad Nacional del Sur \\ Correo electrónico: florencia.pedroni@uns.edu.ar
}

\begin{abstract}
RESUMEN
El objetivo del presente trabajo es realizar un análisis preliminar de la teoría de fijación de precios por arbitraje en el mercado de capitales argentino, haciendo especial hincapié en la inflación como variable de interés para un país emergente, un mercado poco desarrollado y un período bajo análisis con tendencias inflacionarias e intervención del mercado cambiario. Para ello se trabaja con un enfoque de base teórico-empírica. A efectos de plantear el modelo formalmente se realiza un abordaje teórico del tema, con base principalmente en artículos científicos vinculados a la relación entre la tasa de rendimiento de activos y la inflación. El análisis empírico está centrado en un estudio econométrico del rendimiento de las acciones de 19 empresas que cotizan en el mercado de capitales argentino entre 2005-2014 y las vinculaciones con variables de interés, en especial macroeconómicas, incluyendo algunas pruebas de control con variables microeconómicas. Los resultados principales son robustos en relación al efecto sobre el rendimiento de las firmas de las variables explicativas: rendimiento libre de riesgo, rendimiento de mercado, efectos temporales de períodos "bisagra" y efectos sectoriales. Sin embargo, los resultados no son determinantes en el efecto de las variables inflación y tipo de cambio sobre el rendimiento de las acciones.
\end{abstract}

Palabras clave: rendimiento, inflación, datos de panel, teoría de fijación de precios por arbitraje.

Clasificación JEL: E31; E44; G32; O16.

MSC2010: 62H86; 91G50; 91G70.

Artículo recibido el 19 de diciembre de 2019 y aceptado el 20 de julio de 2020. 


\title{
Inflation and returns in emerging markets: the case of Argentina
}

\begin{abstract}
This paper aims to carry out a preliminary analysis of the arbitrage pricing theory in Argentine capital market, with special emphasis on inflation as a variable of interest for an emerging country, an underdeveloped financial market, and a period under analysis with inflationary trends and exchange market intervention. To that end, we work with a theoretical-empirical method. In order to formulate the model, we make a theoretical approach to the subject, based mainly on scientific articles that examine the relationship between the rate of return on assets and inflation. We focus our empirical analysis in an econometric study of the shares' performance of 19 companies listed on Argentinian capital market in the 2005-2014 period, and the associations with variables of interest, especially macroeconomic ones, also including some control tests with microeconomic variables. The main findings are robust about the effect on firms' performance of the following explanatory variables: risk-free interest rate, market performance, temporary effects of "hinge" periods and sector effects. However, the results are not determinant in reference to the effect of the inflation and the exchange rate variables on stocks' performance.
\end{abstract}

Keywords: yield, inflation, panel data, arbitrage pricing theory.

JEL classification: E31; E44; G32; O16.

MSC2010: 62H86; 91G50; 91G70.

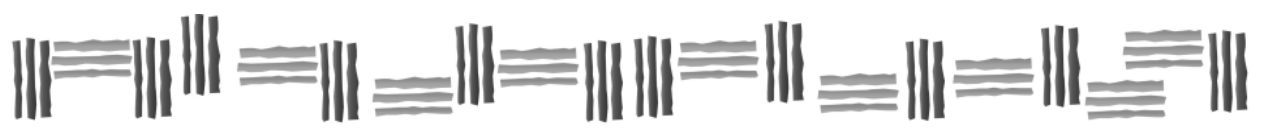




\section{Introducción.}

Si bien existe una innumerable cantidad de estudios que analizan los factores de influencia sobre el rendimiento requerido de acciones, la mayoría de ellos se basa en información de mercados desarrollados, sobre todo centrados en Estados Unidos, en los años posteriores a la Segunda Guerra Mundial (en adelante, periodo posbélico). Algunas investigaciones más recientes reexaminan resultados de esas épocas, realizando estudios empíricos comparativos entre países o intentando fundamentar hallazgos empíricos que contradicen la teoría económica (por ejemplo, la refutación del efecto de Fisher).

En países subdesarrollados, donde predominan mercados de capitales emergentes, concentrados, con poco volumen de negociación y escasez de información, los estudios son incipientes. Resulta interesante estudiar el contexto en estas economías, pues difiere del entorno de los estudios clásicos sobre la temática, en especial debido a factores macroeconómicos, como el nivel de inflación, las tasas de interés real negativas y la inestabilidad política e institucional. En particular, en el mercado de capitales argentino el comportamiento de las acciones en contextos inflacionarios ha sido relativamente poco estudiado. Actualmente, el análisis del caso argentino se torna aún más relevante por situaciones inusuales como las restricciones sobre el mercado de divisas e indirectamente sobre el mercado inmobiliario, que agudizan los problemas de inversión y limitan las alternativas tradicionales (Guerra, 2012).

En el marco del interés en esta problemática, se plantea realizar una investigación para identificar los factores macroeconómicos vinculados con el rendimiento requerido de un conjunto de acciones en el mercado de capitales argentinos, a través de un modelo tipo fijación de precios por arbitraje, haciendo especial hincapié en la inflación. Este artículo se desarrolla en el marco de un proyecto de investigación más amplio, cuyo objeto de estudio se centra en las metodologías de valoración de empresas en mercados emergentes y contextos inflacionarios.

Para el desarrollo del trabajo, se propone un tratamiento de la temática mediante una investigación descriptiva cuantitativa (correlacional) y en la última etapa, explicativa. Para ello se trabaja con un enfoque de base teórico-empírica. A efectos de plantear el modelo formalmente se realiza un abordaje teórico del tema, con base principalmente en artículos científicos vinculados a la relación entre la tasa de rendimiento de activos y la inflación. El análisis empírico está centrado en el estudio del rendimiento de las acciones de 19 empresas que cotizan en el mercado de capitales argentino durante los últimos 10 años y las vinculaciones con variables de interés, en especial macroeconómicas (como la inflación, el tipo de cambio, el producto bruto interno, el rendimiento del mercado, etc.), con algunas pruebas de control con variables microeconómicas (como el sector, el tamaño, entre otras).

Según Swoboda (2002) es notoria la escasez que existe en Argentina de estudios empíricos que utilicen las principales teorías sobre la formación de los precios de los activos financieros. En ese sentido, la presente investigación se considera de relevancia por integrar el reducido grupo de trabajos que aplican el modelo de fijación de precios por arbitraje al mercado de capitales argentino, junto con los documentos de Raele (2011) y Swoboda (2002). En el caso de Raele (2011), emplea el mencionado método de valoración usando datos de cuarto trimestre de 2009 para las 14 empresas que conforman el Merval a esa fecha; mientras que Swoboda (2002) lo utiliza para calcular los rendimientos de 16 especies del panel líder del Mercado de Valores de Buenos Aires durante el lapso 1995-2000.

Asimismo, este artículo complementa y extiende los avances de algunos estudios que examinan la relación entre rendimientos del mercado de acciones y tasa de inflación en Argentina. En primer lugar, este documento actualiza las estimaciones de Choudhry (2001) estudiando el fenómeno en un período posterior. En segunda instancia, a diferencia de los trabajos de Guerra (2012) y Dapena 
(2013), esta investigación utiliza el rendimiento accionario individual como variable dependiente (en lugar de índices de mercados) y estudia el efecto de la inflación a partir de más de un indicador (específicamente se emplean cinco proxies para aproximar la variable de interés). Adicionalmente, se extiende el estudio de Dapena (2013) al efectuarse estimaciones multivariadas y no solo análisis de correlación.

El trabajo se estructura de la siguiente manera. En la sección 2 se presenta el marco conceptual, integrado por los modelos teóricos vinculados al tema (subsección 2.1) y los antecedentes empíricos en la relación entre rendimientos e inflación (subsección 2.2). En el apartado 3 se detalla el abordaje metodológico, las fuentes de información, las variables de interés y los métodos estadísticos y econométricos utilizados para el análisis empírico. En la sección 4 se exhiben los resultados, en primer lugar, de las tendencias y correlaciones de las series y a continuación los análisis econométricos. Por último, se concluye sobre el estudio, sus limitaciones y los futuros avances en la investigación.

\section{Marco conceptual y de antecedentes.}

\subsection{Modelos teóricos.}

Existen diversos modelos de valoración de activos financieros. El más tradicional, CAPM (de sus siglas en inglés, Capital Asset Princing Model) indica que el rendimiento requerido de un activo está explicado por el coeficiente de riesgo sistemático presentado por el mismo, asociado con su covarianza con la prima de riesgo de mercado. Luego se desarrollaron otros modelos, como el modelo de mercado o el modelo de los tres factores (Fama \& French, 1993). En este trabajo, se centra la atención particularmente en el modelo de fijación de precios por arbitraje o APT (por sus siglas en inglés, Arbitrage Pricing Theory), desarrollado por Ross (1976).

El modelo de fijación de precios por arbitraje plantea que el retorno esperado de un activo financiero puede ser modelado como una función lineal de varios factores correlacionados (ecuación 1), habitualmente macroeconómicos, donde la sensibilidad a cambios en cada componente es representada por un factor específico.

$$
\widetilde{R_{\iota}}=E_{i}+\beta_{i 1} * \tilde{\delta}_{1}+\beta_{i 2} * \tilde{\delta}_{2}+\cdots+\beta_{i k} * \tilde{\delta}_{k}+\tilde{\varepsilon}_{i}
$$

donde $\widetilde{R_{l}}$ es el rendimiento esperado de cada activo, $\delta$ son los factores que pueden explicar el rendimiento (es decir, que se encuentran correlacionados con el mismo) y $\beta$ las sensibilidades ante cambios de cada variable explicativa.

APT es un modelo multifactorial derivado de análisis empíricos, mientras que el tradicional modelo CAPM es un modelo de valoración teórico de equilibrio. Debido a esta diferencia, el modelo APT prescinde de varios supuestos sobre los que se basa CAPM, pero por el mismo motivo, las predicciones son un poco más débiles.

Dado que se trata de un modelo de naturaleza empírica, es esperable que el número y el entorno de los factores no sea constante en el tiempo ni para diferentes economías. El impacto de los determinantes sobre el precio del activo se manifiesta en sus movimientos imprevistos y debe representar influencias no diversificables. Habitualmente se utilizan variables tales como el nivel de crecimiento económico, el desarrollo industrial, las tasas de interés y la inflación. Diversos autores examinan empíricamente el modelo APT, encontrando varios factores que explican el rendimiento de un activo en diferentes épocas y mercados (Roll \& Ross, 1980; Chen \& Ingersoll, 1983; Burmeister \& Wall, 1986; entre otros). 
Este trabajo procura en particular detectar factores comunes para empresas de capital abierto, haciendo especial hincapié en el factor inflacionario, debido a la naturaleza de la economía argentina en los últimos años. Al respecto, desde una perspectiva teórica es válido mencionar que la paridad de Fisher define el tipo de interés real ex-ante a un determinado plazo como la diferencia entre el tipo de interés nominal a dicho plazo y la tasa de inflación esperada (ecuación 2).

$$
\left(1+i_{t}^{N}\right)=\left(1+i_{t}^{R}\right)\left(1+\pi_{t}\right), \text { o equivalentemente, } i_{t}^{N}=i_{t}^{R}+\pi_{t}+\pi_{t} \times i_{t}^{R}
$$

donde $i_{t}^{N}$ es el tipo de interés nominal, $i_{t}^{R}$ el tipo de interés real y $\pi_{t}$ la inflación esperada, todas para un período t. El cumplimiento de la hipótesis de Fisher a largo plazo supone que el tipo de interés nominal y la tasa de inflación presentan la misma tendencia, lo que implica que el tipo de interés real es estacionario y el tipo nominal podría ser predictor de las expectativas de inflación.

Siguiendo a Fornero (2003), en las situaciones en que existe incertidumbre acerca de la magnitud de la inflación, se suele considerar que hay tres posibles relaciones entre la tasa nominal y la tasa de inflación: 1) ilusión monetaria: no se incluyen los cambios de la inflación en las tasas nominales; 2) adaptación con retardo (adaptive lag): hay un retraso en el cambio de la tasa nominal frente a la aceleración o la desaceleración de la inflación; 3) expectativas racionales: la tasa nominal incorpora inmediatamente los cambios en la inflación esperada.

Asimismo, los tipos de interés reales son iguales en todos los países cuando los mercados están en equilibrio, por lo que las diferencias en los tipos de interés nominales reflejan variaciones en la tasa de inflación esperada (ecuación 3). Esta relación es conocida como Efecto Internacional de Fisher.

$$
\frac{1+i_{A}}{1+i_{B}}=\frac{1+\pi_{A(\$)}}{1+\pi_{B(U S)}}
$$

donde $i_{A}$ es el tipo de interés nominal en el país A; $i_{B}$ es el tipo de interés nominal en el país B; $\pi_{A(\$)}$ la inflación del país A, nominada en su moneda local, por ejemplo, en pesos (\$); y $\pi_{B(U S \$)}$ es la inflación del país B, nominada en su moneda local, por ejemplo, en dólares (US\$).

Dado que en este trabajo se utilizan tasas de rendimiento nominal, se prevé que la inflación sea una potencial variable explicativa dentro del grupo de factores macroeconómicos. Al respecto, existe bastante literatura que analiza la relación existente entre la inflación y el precio de las acciones, siendo variados los efectos y vínculos encontrados entre ambos. Según Dapena (2013), entendiendo que las acciones representan derechos de los accionistas sobre los bienes residuales de una firma, que los mismos pueden ser bienes reales y que el precio de las acciones refleja la posibilidad futura de la firma de ajustar sus ingresos como consecuencia de las variaciones de precios (en menor o mayor medida), un enfoque propone que los títulos de capital pueden ajustar su precio acorde a la inflación y por ende proteger al ahorrista. Sin embargo, otra postura sostiene que la inflación puede afectar negativamente el precio de las acciones, al erosionar los márgenes de ganancias de las empresas (suben los costos más que los ingresos), al reducir las perspectivas de crecimiento de la economía (y por ende las ganancias de las empresas por efecto agregado de la inflación) y al incrementar la tasa nominal de descuento. A continuación, se analiza la evidencia empírica vinculada a la relación entre rendimiento e inflación a nivel internacional y nacional.

\subsection{Antecedentes empíricos.}

En esta sección se describen los principales estudios empíricos que examinan la relación entre rendimiento accionario e inflación, organizados del siguiente modo. En primer lugar, se exponen las investigaciones realizadas en mercados desarrollados, principalmente en los Estados Unidos (EEUU). A continuación, se presentan las pesquisas con abordaje internacional, efectuadas incluyendo 
mercados de diferentes países. En última instancia, se detallan los trabajos realizados sobre mercados emergentes, en contextos de alta inflación. Los estudios referentes a mercados desarrollados pueden asimismo ordenarse en tres subgrupos según el periodo al cual se refieren los datos: (i) 1953-1972 (Bodie, 1976; Nelson, 1976; Jaffe \& Mandelker, 1976; Fama \& Schwert, 1977); (ii) 1953-2000 (Fama, 1981; Kaul, 1987; Marshall, 1992; Balduzzi, 1995; Hagmann \& Lenz, 2004) y (iii) 1990-2012 (Ang, Brière \& Signori, 2012; Ciner, 2015).

\section{Mercados desarrollados - subgrupo 1}

Existen diversas acepciones de cuándo se considera que un activo financiero actúa como cobertura contra la inflación. Una de ellas establece que un título es una protección si y solo si su rendimiento real es independiente de la tasa de inflación, lo que implica que ceteris paribus un cambio en la tasa de inflación debería ir acompañado de una variación similar en la tasa nominal de rendimiento sobre el capital. Esto se expresa más comúnmente como una correlación positiva entre la tasa de rendimiento nominal y la tasa de inflación (Branch, 1974; Fama \& MacBeth, 1974; Oudet, 1973). Por su parte, otra postura plantea que tal cobertura existe si el activo elimina o al menos reduce la posibilidad de que su tasa de rendimiento real caiga por debajo de un valor "piso" determinado, como puede ser cero (Reilly, Johnson \& Smith, 1970; Johnson, Reilly \& Smith, 1971; Cagan, 1974). Basado en este último concepto, Bodie (1976) intenta determinar en qué medida las acciones ordinarias son una cobertura contra la inflación empleando a tal fin datos anuales, trimestrales y mensuales del mercado estadounidense para el período 1953-1972. Los resultados obtenidos de las tres regresiones parecen indicar que, contrariamente a la creencia común entre los economistas, la rentabilidad real de las acciones se relaciona negativamente tanto la inflación anticipada como con la no anticipada, al menos en el corto plazo. Esta correlación negativa lleva a la conclusión sorprendente y un tanto inquietante que, al utilizar las acciones ordinarias como una cobertura contra la inflación, éstas deben venderse a corto plazo. A similares conclusiones aborda Nelson (1976), quien trabaja sobre el mismo mercado y período, pero con datos mensuales. Sus resultados son consistentes con la hipótesis de que el mercado responde inversamente a los cambios contemporáneos en las expectativas de inflación. Según el autor, si la evidencia sugiere que tanto los rendimientos ex ante como ex post están correlacionados con ratios de cambios (actuales y pasados) en el Índice de Precio al Consumidor (IPC), entonces es posible usar la información de las series de IPC para predecir los rendimientos futuros de las acciones.

Jaffe y Mandelker (1976) extienden las fronteras de sus predecesores: analizan no sólo datos mensuales del lapso temporal 1953-1971, sino que además efectúan regresiones con observaciones anuales para un intervalo más amplio. Encuentran una relación negativa entre los rendimientos y las tasas de inflación concurrentes para 1953-1971 y un vínculo positivo entre las dos variables para un período de tiempo de mayor longitud (1875-1970). Al distinguir entre inflación esperada y no esperada los hallazgos son divergentes. Para el período 1953-1971, los rendimientos de las acciones presentan una relación negativa y significativa con la tasa esperada de inflación (inconsistencia con hipótesis de Fisher y posible ineficiencia de mercado). Sin embargo, para el período 1875-1970 los retornos accionarios anuales aparecen como independientes de la inflación pasada. Finalmente, y de modo consistente con estudios previos, sus resultados también sugieren un vínculo negativo entre inflación no esperada y rendimiento.

La investigación de Fama y Schwert (1977), desarrollada sobre el mismo período que estudios anteriores (1953-1971), amplía al espectro de análisis. Esto es, estima si diferentes activos actúan como cobertura contra la inflación (esperada y no esperada). Los autores encuentran que sólo la posesión de inmuebles es una protección completa contra la inflación (esperada y no esperada). Los bonos y letras del gobierno norteamericano ofrecen una cobertura completa contra la inflación esperada. Los ingresos laborales muestran una pequeña relación a corto plazo con la inflación (esperada y no esperada). El resultado más anómalo es que el rendimiento de las acciones comunes 
está negativamente correlacionado con el componente esperado de la tasa de inflación y, probablemente, también con el componente no anticipado.

\section{Mercados desarrollados - subgrupo 2}

El segundo subgrupo de estudios en mercados desarrollados, además de testear empíricamente las relaciones entre inflación y rendimiento accionario, intenta explicar a través de diferentes teorías las aparentes inconsistencias de la evidencia posbélica con las predicciones de la teoría económica. En este orden de ideas, Fama (1981) esboza y testea la "hipótesis del efecto proxy", una unión de modelos de expectativas racionales para los sectores monetario y real. Según esta hipótesis, las relaciones positivas entre los retornos de los títulos y la actividad real (proveniente del sector real) se combinan con las relaciones negativas entre la inflación y la actividad real (procedentes del sector monetario) para inducir relaciones negativas espurias entre los retornos de las acciones y la inflación. Sus explicaciones se sustentan en la combinación de la teoría de la demanda de dinero y la teoría cuantitativa del dinero. De este modo, la relación anómala negativa entre el retorno accionario e inflación desaparecerá cuando tanto las variables reales como las medidas de inflación esperada e inesperada se utilicen para explicar los rendimientos de las acciones.

Kaul (1987) supone que la relación entre los rendimientos de las acciones y la inflación es causada por el proceso de equilibrio en el sector monetario. Asimismo, plantea que estas relaciones varían con el tiempo en una de manera sistemática influenciadas por factores vinculados a la oferta y la demanda de dinero. Según Kaul (1987), la hipótesis proxy de Fama (1981) asume que la oferta monetaria se determina exógenamente (independiente del nivel de la actividad real) cuando un modelo completo del sector monetario debiera considerar también la respuesta de las autoridades monetarias, es decir, el proceso de oferta de dinero. Usando datos de los Estados Unidos, Canadá, el Reino Unido y Alemania encuentra para el período 1952-1983 que las relaciones negativas entre el retorno accionario y la inflación son causadas por la demanda de dinero y por los efectos contracíclico de la oferta de dinero. Por otra parte, los movimientos procíclicos en el dinero, la inflación, y los precios de las acciones durante los 1930 conducen a relaciones que son positivas o insignificantes.

El modelo equilibrado de fijación de precios de activos monetarios de Marshall (1992) implica correlaciones negativas entre rendimientos esperados de los activos e inflación anticipada. Asimismo, predice que tal correlación es más fuertemente negativa cuando la inflación se genera por las fluctuaciones de la actividad económica real, respecto a cuando se origina en variaciones monetarias. El modelo, testado sobre datos trimestrales del mercado estadounidense para el período 1959-1990, es coherente tanto con una respuesta negativa como positiva de la rentabilidad de las acciones a shocks inflacionarios, pues la principal fuente de las variaciones inflacionarias son las fluctuaciones la actividad económica real.

Balduzzi (1995) reexamina la hipótesis de proxy de Fama (1981) como la principal explicación de la correlación negativa entre los rendimientos de las acciones y la inflación. Emplea un conjunto de datos trimestrales sobre: el crecimiento de la producción industrial, el crecimiento de la base monetaria, la inflación, las tasas de las letras del tesoro a 3 meses y la rentabilidad ponderada del índice NYSE para los períodos 1954-1976 y 1977-1990. Encuentra que el crecimiento de producción induce solamente una débil correlación negativa entre la inflación y el rendimiento, siendo la inflación per se responsable de la mayor parte de la interacción dinámica con los retornos accionarios. Asimismo, argumenta que la tasa de interés contribuye a una parte sustancial de la correlación negativa entre los retornos y la inflación.

Hagmann y Lenz (2004) reexaminan la relación empírica entre rendimiento real y diferentes componentes de la inflación: (i) inflación ex post; (ii) inflación esperada; (iii) cambios en la inflación esperada y (iv) inflación no anticipada. Usando la metodología VAR (vector de auto regresión) se propone una descomposición de dichas series en componentes económicamente interpretables 
conducidos mediante la oferta agregada, la demanda real y los shocks del mercado monetario. Los resultados empíricos soportan la hipótesis proxy de Fama (1981) y las predicciones de varios modelos generales de equilibrio. El signo de la correlación entre el rendimiento real y la inflación (no anticipada) depende del origen del shock: es negativo para shocks originados en la oferta agregada y en la demanda real, pero es positivo para los shocks monetarios. Asimismo, se encuentra que la correlación entre el rendimiento real esperado y la inflación anticipada es independiente del origen del shock estructural, lo que es ampliamente consistente con los resultados obtenidos por Marshall (1992). El estudio también muestra que ambas relaciones (entre el rendimiento real o interés real y la inflación) han decrecido desde los años 80, principalmente debido a una disminución de los shocks estructurales en la economía real.

\section{Mercados desarrollados - subgrupo 3}

La tercera subcategoría de trabajos en mercados desarrollados incluye investigaciones efectuadas con información de las últimas dos décadas. Sus resultados presentan particularidades interesantes (relaciones positivas, análisis de rendimientos accionarios individuales, distinciones sectoriales) respecto a estudios empíricos precedentes. Ang, Brière y Signori (2012) estudian la capacidad de las acciones individuales para actuar como cobertura frente a la inflación. Consideran que existen buenas razones para analizar el rendimiento de acciones individuales y no de índices de mercado. En primer lugar, y lo más importante, la construcción de portafolios basados en acciones individuales cuyos rendimientos covarían fuertemente con la inflación tiene el potencial de proporcionar una mejor protección contra la inflación que el índice de rendimiento de un conjunto de títulos. En segunda instancia, existe una heterogeneidad considerable entre las empresas; y como tercer fundamento, el examen de las acciones individuales también permite investigar qué tipos de acciones o sectores son mejores salvaguardas respecto a otros. Los resultados demuestran que, si bien la correlación del mercado global con la inflación es negativa, hay un subconjunto significativo de acciones con betas inflacionarios altos y significativamente positivos a lo largo de la muestra. Al clasificar las carteras accionarias en quintiles (con base en betas inflacionarios realizados ex post) encuentran que la cartera quintil con los betas más altos ha tenido una correlación positiva con la inflación. Este quintil se integra mayoritariamente por acciones de los sectores de petróleo, gas y tecnología. Las carteras quintiles restantes tienen betas de inflación negativos. Por lo tanto, un subconjunto no despreciable de las acciones ha covariado positivamente con la inflación.

Por su parte, Ciner (2015) emplea un método de descomposición de frecuencia y examina si existe una dependencia persistente en el vínculo inflación-rendimiento, lo que implica que shocks inflacionarios de alta y baja frecuencia tienen diferente efecto sobre los movimientos de precios de las acciones. Además, explora el vínculo retorno/inflación basándose en carteras de acciones de empresas industriales, a fin de determinar si ciertos sectores del mercado accionario pueden ser considerados una mejor cobertura frente a la inflación. Supone que si las acciones son una buena cobertura ante la inflación entonces debiera existir una relación positiva entre su rendimiento y la inflación no anticipada. Los resultados del estudio soportan la hipótesis principal: la relación entre rendimiento de la acción e inflación depende de la persistencia de los shocks inflacionarios. Shocks de largo plazo de tendencia muestran betas negativos, en concordancia con estudios anteriores, mientras que los betas de los shocks de inflación no anticipada son positivos. Análogamente a lo encontrado por Ang, Brière y Signori (2012), dichos betas positivos se encuentran en sectores vinculados a commodities (carbón, mineral, combustible, oro, agricultura) e industrias relativas a la tecnología (teléfonos, software, chips).

\section{Evidencia comparativa transnacional}

Considerando la evidencia empírica existente, principalmente referida al mercado estadounidense, respecto a la relación negativa entre inflación y rendimiento, comienzan a surgir una serie de investigaciones de abordaje internacional, con el objeto de dilucidar si los resultados mencionados se 
mantienen en contextos diferentes. En este sentido Nichols (1976), por ejemplo, propone llevar adelante las investigaciones en países de alta inflación.

Cagan (1974) estudia la relación rendimiento/inflación sobre varios países, incluyendo los períodos pre, inter y posbélicos. En general, sus resultados revelan que las acciones comunes representadas en los índices examinados mantienen, en promedio, su valor real a lo largo de épocas de depreciación monetaria, excepto en los casos de hiperinflación o devastación bélica. Esto es, un importante grupo de acciones ofrece protección contra la inflación sólo para tenencias de largo plazo, en momentos del tiempo que no se corresponden con tales situaciones anómalas.

Branch (1974) estudia si las acciones son una cobertura contra la inflación a nivel internacional para el período 1953-1969. A tal fin, emplea datos de 22 países con diferentes contextos (desarrollados y subdesarrollados): Austria, Canadá, Dinamarca, Finlandia, Francia, Italia, Japón, Países Bajos, Nueva Zelanda, Noruega, Sudáfrica, Suecia, Reino Unido, EEUU, Irlanda, Portugal, España, Chile, Colombia, México y Perú. Los resultados indican que las acciones son una protección parcial, y no completa, contra la inflación en el largo plazo.

Firth (1979) examina la relación inflación y rendimiento de las acciones comunes en el contexto británico, considerando periodos de tiempo de diferente extensión: datos anuales de 19191976 y mensuales entre 1955 y 1976. Parte de la evidencia soporta la hipótesis de Fisher y contrasta los resultados de estudios anteriores. Con base en las observaciones mensuales para el lapso 19551976, los coeficientes de regresión son positivos (al nivel de 0,05), en línea con el "efecto Fisher" y en contraste con los coeficientes significativamente negativos reportados por Jaffe y Mandelker (1976) y Nelson (1976). Asimismo, excepto por un subperíodo, todos los coeficientes son mayores que la unidad, indicando que los inversores son más que recompensados por la tasa esperada de inflación. Las regresiones estimadas empleando datos anuales entre 1919-1976 nuevamente proveen cierta evidencia a la hipótesis de Fisher: los betas son cercanos a cero y algunos no resultan significativos estadísticamente. Por ende, no se encuentra relación negativa significativa (fuerte) entre rendimiento e inflación.

Solnik (1983) indaga la relación entre las expectativas de inflación y los precios de activos para los principales mercados de valores: EEUU, Japón, UK, Suiza, Francia, Alemania, Canadá, Países Bajos, durante la década de los 70 (1971-1980). Sus resultados rechazan profundamente el supuesto fisheriano, según el cual los rendimientos reales son independientes de las expectativas inflacionarias. Usando las tasas de interés como un proxy para la inflación esperada, los datos proporcionan un soporte consistente para el modelo Geske y Roll (1983) cuya hipótesis básica es que los movimientos de precios de las acciones señalan revisiones (negativas) en las expectativas inflacionarias. Finalmente, un débil efecto de tasa de interés real se encuentra para algunos países.

Gultekin (1983) investiga la relación entre el rendimiento de las acciones comunes y la inflación en veintiséis países para el período de posguerra. Para ello prueba las hipótesis de Fisher según las que las tasas reales de rentabilidad de las acciones ordinarias y las tasas de inflación esperada son independientes y que los rendimientos de las acciones nominales varían uno-a-uno en correspondencia con la inflación esperada. Usando regresiones de series de tiempo, no encuentra una relación positiva fiable entre rendimientos accionarios nominales y las tasas de inflación para el período 1947-1979. Los coeficientes de regresión son predominantemente negativos. Por otra parte, los resultados revelan que la relación retorno accionario/inflación no es estable en el tiempo y que hay diferencias entre los países. También observa que países con mayores tasas de inflación tienen en general mayores rendimientos nominales; mientras que las tasas de retorno reales han disminuido en la mayoría de los países desde mediados de la década de 1960. 


\section{Investigaciones en mercado emergentes con contextos de alta inflación}

Una visión sintética de los trabajos hasta aquí descriptos, permite notar que la extensión del análisis más allá del contexto estadounidense muestra resultados diversos e incluso contrastantes con la abultada literatura, encontrándose cierta evidencia a favor de la hipótesis de Fisher. El último grupo de estudios empíricos que se presenta a continuación se enfoca en países con alta inflación, siendo uno de ellos específico del caso argentino.

Choudhry (2001) investiga la relación a corto plazo entre el rendimiento de las acciones y la inflación en cuatro países de alta inflación (latinoamericanos y centroamericanos): Argentina, Chile, México y Venezuela, empleando datos mensuales del período 1981-1998. Analiza rendimiento nominal y real y para cada uno de ellos efectúa 4 regresiones (considerando diferente cantidad de rezagos y proyecciones). Los resultados, en comparación con el grueso de la investigación anterior (realizada en contextos de baja inflación), proporcionan evidencias interesantes.

La evidencia empírica en relación a los rendimientos nominales, indica que el efecto Fisher es posible para los activos de más riesgo en el corto plazo bajo condiciones de alta inflación. Se observa una relación positiva de uno a uno entre el retorno e inflación (actuales). Esto implica que las acciones son una buena cobertura contra las altas las tasas de inflación. También se encuentra una relación positiva entre rendimiento actual y tasa de inflación de un período anterior. Este resultado concuerda con Nelson (1976) quien afirma que la relación entre los rendimientos nominales actuales y la tasa de inflación un período rezagada debe ser directa debido a la correlación positiva entre la inflación pasada y esperada. Los resultados muestran, además, que los rezagos más antiguos de la inflación también influyen en la tasa actual de rendimiento, indicando que tasas pasadas de inflación contienen información con respecto a la tasa de inflación futura. Se encuentra poca evidencia referente a los efectos de las proyecciones inflacionarias sobre el rendimiento de las acciones, lo que revela que la inflación futura puede no contener mucha información.

Empleando retornos reales, se encuentra cierta evidencia de una relación inversa entre los rendimientos reales actuales y la inflación actual y un período rezagada. Estos resultados respaldan la ausencia del efecto Fisher y proporcionan evidencia de la teoría de Lintner. Se obtiene poca evidencia sobre el efecto de la inflación un período rezagado y se esbozan algunos de los efectos de las tasas de inflación pasadas y futuras. Los resultados presentados en este artículo abogan por una mayor investigación en este campo.

Guerra (2012) desarrolla un análisis econométrico de las relaciones de corto y de largo plazo entre el rendimiento de las acciones y la tasa de inflación en Argentina para el período comprendido entre enero de 1993 y abril de 2013. Como medida de rendimiento accionario emplea el logaritmo de la tasa de variación mensual del Índice Merval y para aproximar la tasa de inflación mensual utiliza la tasa de cambio logarítmica del IPC. En concordancia con lo expuesto por las investigaciones empíricas realizadas para otros países, se encuentra evidencia de la existencia de una relación negativa de corto plazo entre el rendimiento de las acciones y la tasa de inflación para el período 2002-2013. No obstante, en lo que se refiere a la muestra correspondiente al período 1993-2001, la reducida precisión de las estimaciones no permite obtener resultados concluyentes. Por otro lado, el estudio de largo plazo no posibilita confirmar la existencia de una relación positiva entre el rendimiento de las acciones y la tasa de inflación, por lo que resulta posible que el crecimiento conjunto del precio de las acciones y la tasa de inflación sea provocado por el componente tendencial de ambas series y no por la existencia de relación entre las variables.

Dapena (2013) analiza lo sucedido en el mercado accionario argentino, donde el nivel de precios o inflación acumulada para el período diciembre 2001 a marzo 2013 se incrementa en un $555 \%$, mientras que el nivel de precio de las acciones medido por el índice Merval aumenta un $832 \%$. La correlación entre la variación en el índice de precios (inflación) y los retornos accionarios es de 
casi cero $(-0,07)$, lo que demuestra que los datos no se encuentran correlacionados contemporáneamente. Los datos evidencian que al menos en el período considerado, la inversión en una canasta diversificada de acciones se ha encontrado por encima de la inflación. De hecho, el rendimiento en el precio de las acciones en equilibrio en el largo plazo debiera encontrarse por encima de la tasa de inflación, ya que debe retribuir también el crecimiento real en los negocios de las empresas y una prima por riesgo.

López (2016) estudia la relación entre inflación y retornos accionarios reales en la Argentina encontrando un vínculo negativo, que parece originarse en el componente inflacionario no esperado. Adicionalmente sus resultados respaldan la hipótesis proxy de Fama en ambos países pues verifican que la actividad económica real se asocia negativamente con la inflación y positivamente con los retornos accionarios.

Lanche-Bonde (2015) examina la relación entre el rendimiento accionario (Índice General de la Bolsa de Comercio de Buenos Aires) y la tasa de inflación en la Argentina verificando que, en el largo plazo, las acciones actúan como cobertura: entre 2002 y 2013 el IPC creció un $175 \%$, mientras que el índice general de la bolsa sufrió un alza del $234 \%$ (variación medida como cociente entre el logaritmo natural [ln] del último año sobre el $\ln$ del primero). La Tabla 1 resume los estudios empíricos detallados precedentemente.

Tabla 1. Resumen de los antecedentes empíricos.

\begin{tabular}{|c|c|c|c|c|c|}
\hline \multirow{2}{*}{$\begin{array}{l}\text { Autor } \\
\text { (año) }\end{array}$} & \multirow{2}{*}{ Objetivo } & \multicolumn{3}{|c|}{ Fuente de información } & \multirow{2}{*}{ Resultados } \\
\hline & & Periodo & Frecuencia & Mercado & \\
\hline $\begin{array}{l}\text { Bodie } \\
(1976)\end{array}$ & \multirow{2}{*}{$\begin{array}{l}\text { Busca determinar en } \\
\text { qué medida las } \\
\text { acciones ordinarias son } \\
\text { una cobertura contra la } \\
\text { inflación }\end{array}$} & $1953-1972$ & $\begin{array}{l}\text { Mensual, } \\
\text { trimestral y } \\
\text { anual }\end{array}$ & EEUU & $\begin{array}{l}\text { Relación negativa entre RR e } \\
\text { inflación (E y NE) en el CP }\end{array}$ \\
\hline $\begin{array}{l}\text { Nelson } \\
(1976)\end{array}$ & & $1953-1972$ & Mensual & EEUU & $\begin{array}{l}\text { Relación negativa entre } \\
\text { rentabilidad e inflación (E y } \\
\text { NE) }\end{array}$ \\
\hline \multirow[t]{2}{*}{$\begin{array}{l}\text { Jaffe \& } \\
\text { Mandelker } \\
\text { (1976) }\end{array}$} & \multirow{2}{*}{$\begin{array}{c}\text { Investiga } \\
\text { empíricamente la } \\
\text { relación entre el } \\
\text { rendimiento de activos } \\
\text { riesgosos (acciones) y } \\
\text { la inflación }\end{array}$} & $1875-1970$ & Anual & \multirow[t]{2}{*}{ EEUU } & \multirow{2}{*}{$\begin{array}{c}\text { Relación inflación y } \\
\text { rendimiento contemporáneos: } \\
\text { negativa para 1953-1971 y } \\
\text { positiva entre 1875-1970. } \\
\text { Inflación E y retorno: relación } \\
\text { negativa durante 1953-1971 e } \\
\text { independientes entre 19875 y } \\
1970 \\
\end{array}$} \\
\hline & & $1953-1971$ & Mensual & & \\
\hline $\begin{array}{l}\text { Fama \& } \\
\text { Schwert } \\
(1977)\end{array}$ & $\begin{array}{l}\text { Estiman si diferentes } \\
\text { activos son una } \\
\text { cobertura contra la } \\
\text { inflación }\end{array}$ & $1953-1971$ & $\begin{array}{l}\text { Mensual, } \\
\text { trimestral y } \\
\text { semestral }\end{array}$ & EEUU & $\begin{array}{l}\text { Relación negativa entre } \\
\text { rendimiento e inflación (E), y } \\
\text { probablemente, con la NE }\end{array}$ \\
\hline $\begin{array}{l}\text { Fama } \\
(1981)\end{array}$ & \multirow{3}{*}{$\begin{array}{l}\text { Intentan explicar las } \\
\text { relaciones anómalas } \\
\text { entre el rendimiento de } \\
\text { las acciones y la } \\
\text { inflación }\end{array}$} & $1953-1977$ & $\begin{array}{l}\text { Mensual, } \\
\text { trimestral y } \\
\text { anual. }\end{array}$ & EEUU & $\begin{array}{l}\text { Relación negativa entre RR e } \\
\text { inflación, explicada mediante } \\
\text { efecto proxy }\end{array}$ \\
\hline $\begin{array}{l}\text { Kaul } \\
(1987)\end{array}$ & & $\begin{array}{l}1926-1940 \\
1952-1983\end{array}$ & $\begin{array}{l}\text { Mensual, } \\
\text { trimestral y } \\
\text { anual. }\end{array}$ & $\begin{array}{l}\text { EEUU, } \\
\text { Canadá, } \\
\text { UK y } \\
\text { Alemania }\end{array}$ & $\begin{array}{c}\text { 1926-1940: relación } \\
\text { insignificante o positiva } \\
\text { 1952-1983: Relación negativa } \\
\text { entre RR e inflación (E y NE) }\end{array}$ \\
\hline $\begin{array}{l}\text { Marshall } \\
(1992)\end{array}$ & & $1959-1990$ & Trimestral & EEUU & $\begin{array}{c}\text { Correlación negativa entre RR } \\
\text { e inflación }\end{array}$ \\
\hline
\end{tabular}




\begin{tabular}{|c|c|c|c|c|c|}
\hline \multirow{2}{*}{$\begin{array}{l}\text { Autor } \\
\text { (año) }\end{array}$} & \multirow{2}{*}{ Objetivo } & \multicolumn{3}{|c|}{ Fuente de información } & \multirow{2}{*}{ Resultados } \\
\hline & & Periodo & Frecuencia & Mercado & \\
\hline $\begin{array}{l}\text { Balduzzi } \\
\text { (1995) }\end{array}$ & $\begin{array}{l}\text { Reexamina hipótesis } \\
\text { proxy de Fama (1981) }\end{array}$ & $\begin{array}{l}1954-1976 \\
1977-1990\end{array}$ & Trimestral & EEUU & $\begin{array}{c}\text { Identifica a la inflación per se } \\
\text { como responsable de la mayor } \\
\text { parte de la interacción } \\
\text { dinámica con los } \\
\text { rendimientos y a la tasa de } \\
\text { interés como contribución } \\
\text { sustancial en la correlación } \\
\text { negativa entre los retornos y } \\
\text { la inflación }\end{array}$ \\
\hline $\begin{array}{l}\text { Hagmann } \\
\text { \& Lenz } \\
\text { (2004) }\end{array}$ & $\begin{array}{l}\text { Reexaminan la } \\
\text { relación empírica entre } \\
\text { en RR y diferentes } \\
\text { componentes de la } \\
\text { inflación: inflación ex- } \\
\text { post; inflación E; } \\
\text { cambios en la inflación } \\
\text { E e inflación NE }\end{array}$ & $1954-2003$ & Trimestral & EEUU & $\begin{array}{l}\text { El signo de la correlación } \\
\text { entre el RR y la inflación } \\
\text { (NE) depende del origen del } \\
\text { shock (negativo para shocks } \\
\text { de variables económicas } \\
\text { reales, positivo en shocks } \\
\text { monetarios). La correlación } \\
\text { entre el RR y la inflación E es } \\
\text { independiente del origen del } \\
\text { shock estructural }\end{array}$ \\
\hline $\begin{array}{l}\text { Ang, } \\
\text { Brière \& } \\
\text { Signori } \\
(2012)\end{array}$ & $\begin{array}{l}\text { Estudian la capacidad } \\
\text { de las acciones } \\
\text { individuales para } \\
\text { actuar como cobertura } \\
\text { frente a la inflación }\end{array}$ & $1989-2010$ & Mensual & EEUU & $\begin{array}{l}\text { Correlación negativa del } \\
\text { rendimiento de mercado con } \\
\text { la inflación. Subconjunto } \\
\text { significativo de acciones con } \\
\text { betas inflacionarios altos y } \\
\text { significativamente positivos } \\
\text { (de los sectores de Petróleo, } \\
\text { Gas y Tecnología) }\end{array}$ \\
\hline $\begin{array}{l}\text { Ciner } \\
(2015)\end{array}$ & $\begin{array}{l}\text { Investiga si relación } \\
\text { entre rendimiento } \\
\text { accionario e inflación } \\
\text { depende de la } \\
\text { persistencia de los } \\
\text { shocks inflacionarios }\end{array}$ & $1990-2012$ & Mensual & EEUU & $\begin{array}{l}\text { Correlación entre rendimiento } \\
\text { e inflación: negativa para } \\
\text { shocks inflacionarios de LP y } \\
\text { positiva para shocks de } \\
\text { inflación NE. Ésta última } \\
\text { correlación positiva en } \\
\text { sectores vinculados a } \\
\text { commodities (carbón, mineral, } \\
\text { combustible, oro, agricultura) } \\
\text { e industrias relativas a la } \\
\text { tecnología (teléfonos, } \\
\text { software) }\end{array}$ \\
\hline $\begin{array}{l}\text { Cagan } \\
(1974)\end{array}$ & $\begin{array}{l}\text { Estudian si las } \\
\text { acciones son una } \\
\text { cobertura contra la }\end{array}$ & 1939-1969 & Anual & $\begin{array}{l}\text { Diferentes } \\
\text { países: } \\
\text { europeos, } \\
\text { norteameri } \\
\text { canos y } \\
\text { asiáticos }\end{array}$ & $\begin{array}{l}\text { Un grupo amplio de acciones } \\
\text { son cobertura contra la } \\
\text { inflación, excepto en } \\
\text { situaciones } \\
\text { hiperinflacionarias o de } \\
\text { devastación post-guerra, pero } \\
\text { no simultáneamente a ellas }\end{array}$ \\
\hline $\begin{array}{l}\text { Branch } \\
(1974)\end{array}$ & $\begin{array}{l}\text { inflacion usando datos } \\
\text { internacionales }\end{array}$ & $1953-1969$ & Anual & $\begin{array}{l}22 \text { países: } \\
\text { europeos, } \\
\text { norteameri } \\
\text { canos } \\
\text { latinomeric } \\
\text { anos }\end{array}$ & $\begin{array}{c}\text { Evidencia de que las acciones } \\
\text { son una protección parcial (y } \\
\text { no completa) contra la } \\
\text { inflación a LP }\end{array}$ \\
\hline
\end{tabular}




\begin{tabular}{|c|c|c|c|c|c|}
\hline \multirow{2}{*}{$\begin{array}{l}\text { Autor } \\
\text { (año) }\end{array}$} & \multirow{2}{*}{ Objetivo } & \multicolumn{3}{|c|}{ Fuente de información } & \multirow{2}{*}{ Resultados } \\
\hline & & Periodo & Frecuencia & Mercado & \\
\hline \multirow[b]{2}{*}{$\begin{array}{l}\text { Firth } \\
(1979)\end{array}$} & \multirow{2}{*}{$\begin{array}{c}\text { Examina la relación } \\
\text { inflación y rendimiento } \\
\text { de las acciones } \\
\text { comunes usando datos } \\
\text { británicos }\end{array}$} & $1955-1976$ & Mensual & & $\begin{array}{l}\text { Parte de la evidencia soporta } \\
\text { la hipótesis de Fisher. No }\end{array}$ \\
\hline & & $1919-1976$ & Anual & Inglaterra & $\begin{array}{l}\text { fuerte entre rendimiento e } \\
\text { inflación: betas cercanos a } \\
\text { cero y algunos no } \\
\text { significativos } \\
\text { estadísticamente }\end{array}$ \\
\hline $\begin{array}{l}\text { Solnik } \\
(1983)\end{array}$ & $\begin{array}{l}\text { Presenta tests de la } \\
\text { relación entre las } \\
\text { expectativas de } \\
\text { inflación y los precios } \\
\text { de activos para los } \\
\text { principales mercados } \\
\text { de valores }\end{array}$ & $1971-1980$ & Mensual & 9 países & $\begin{array}{l}\text { Los movimientos de precios } \\
\text { de acciones señalan } \\
\text { revisiones (negativas) en las } \\
\text { expectativas inflacionarias. } \\
\text { Débil efecto de tasa de interés } \\
\text { real para algunos de estos } \\
\text { países (Japón, Alemania, } \\
\text { Suiza, Francia) }\end{array}$ \\
\hline $\begin{array}{l}\text { Gultekin } \\
\text { (1983) }\end{array}$ & $\begin{array}{l}\text { Investiga } \\
\text { empíricamente la } \\
\text { relación entre el } \\
\text { rendimiento de las } \\
\text { acciones comunes y la } \\
\text { inflación }\end{array}$ & $1947-1979$ & Mensual & 26 países & $\begin{array}{c}\text { No encuentran relación } \\
\text { positiva fiable entre } \\
\text { rendimientos nominales de las } \\
\text { acciones e inflación. } \\
\text { Determinan que la relación } \\
\text { retorno accionario/inflación } \\
\text { no es estable en el tiempo y } \\
\text { que hay diferencias entre los } \\
\text { países (países con mayor } \\
\text { inflación, presentan mayores } \\
\text { rendimientos nominales) }\end{array}$ \\
\hline $\begin{array}{l}\text { Choudhry } \\
\text { (2001) }\end{array}$ & $\begin{array}{l}\text { Investiga la relación a } \\
\text { CP entre el } \\
\text { rendimiento accionario } \\
\text { (nominal y real) y la } \\
\text { inflación E, en países } \\
\text { de alta inflación }\end{array}$ & $\begin{array}{l}\text { 1981-1998 } \\
\text { (Argentina, } \\
\text { México, } \\
\text { Chile) } \\
\text { 1985-1998 } \\
\text { (Venezuela } \\
\text { ) }\end{array}$ & Mensual & $\begin{array}{l}4 \text { países: } \\
\text { Argentina, } \\
\text { México, } \\
\text { Venezuela, } \\
\text { Chile }\end{array}$ & $\begin{array}{c}\text { Relación positiva entre } \\
\text { rendimiento actual e inflación } \\
\text { actual (activos actúan como } \\
\text { cobertura). Inflación pasada } \\
\text { influye en la tasa actual de } \\
\text { rendimiento. Cierta evidencia } \\
\text { de una relación inversa entre } \\
\text { los RR actuales y la inflación } \\
\text { actual y un período rezagada }\end{array}$ \\
\hline $\begin{array}{l}\text { Guerra } \\
(2012)\end{array}$ & $\begin{array}{l}\text { Analiza } \\
\text { empíricamente la } \\
\text { relación entre la tasa } \\
\text { de inflación y el } \\
\text { rendimiento nominal } \\
\text { de las acciones }\end{array}$ & $1993-2013$ & Mensual & Argentina & $\begin{array}{l}\text { Relación negativa de CP entre } \\
\text { el rendimiento e inflación } \\
\text { durante 2002-2013. Para } \\
\text { 1993-2001 las estimaciones } \\
\text { no son concluyentes. Estudio } \\
\text { de LP no permite confirmar } \\
\text { una relación rendimiento - } \\
\text { inflación positiva }\end{array}$ \\
\hline $\begin{array}{l}\text { Dapena } \\
(2013)\end{array}$ & $\begin{array}{l}\text { Analiza la correlación } \\
\text { entre los precios de la } \\
\text { acciones y e inflación }\end{array}$ & $2003-2013$ & Mensual & Argentina & $\begin{array}{l}\text { Relación positiva entre el } \\
\text { rendimiento de una canasta } \\
\text { diversificada de acciones y la } \\
\text { inflación } \\
\end{array}$ \\
\hline $\begin{array}{l}\text { Lanche- } \\
\text { Bode } \\
(2015)\end{array}$ & $\begin{array}{c}\text { Describe el } \\
\text { comportamiento del } \\
\text { mercado de capitales } \\
\text { como fuente de } \\
\text { financiamiento y } \\
\text { resguardo contra la } \\
\text { inflación }\end{array}$ & $1975-2013$ & Anual & Argentina & $\begin{array}{l}\text { Relación positive entre } \\
\text { rendimientos accionarios e } \\
\text { inflación: las acciones son una } \\
\text { cobertura contra la pérdida de } \\
\text { poder adquisitivo }\end{array}$ \\
\hline
\end{tabular}




\begin{tabular}{|c|c|c|c|c|c|}
\hline \multirow{2}{*}{$\begin{array}{l}\text { Autor } \\
\text { (año) }\end{array}$} & \multirow{2}{*}{ Objetivo } & \multicolumn{3}{|c|}{ Fuente de información } & \multirow{2}{*}{ Resultados } \\
\hline & & Periodo & Frecuencia & Mercado & \\
\hline $\begin{array}{l}\text { López } \\
\text { (2016) }\end{array}$ & $\begin{array}{l}\text { Examina la relación } \\
\text { entre retornos } \\
\text { accionarios e inflación } \\
\text { en Argentina y los } \\
\text { compara con Brasil }\end{array}$ & $2002-2014$ & Mensual & $\begin{array}{c}\text { Argentina } \\
\text { y Brasil }\end{array}$ & $\begin{array}{l}\text { Relación negativa entre } \\
\text { inflación y retornos } \\
\text { accionarios reales, } \\
\text { fundamentalmente originada } \\
\text { en la inflación NE }\end{array}$ \\
\hline
\end{tabular}

Fuente: Elaboración propia.

\section{Metodología.}

El objeto de estudio se centra en las metodologías de valoración de empresas en mercados emergentes y contextos inflacionarios, a través de un modelo tipo APT. Se busca identificar qué factores macroeconómicos se vinculan con el rendimiento requerido de un conjunto de acciones en el mercado de capitales argentinos, haciendo especial hincapié en la inflación. Se propone un tratamiento de la temática en el marco de una investigación descriptiva cuantitativa (correlacional) y en la última etapa, explicativa. Para ello se trabaja con un enfoque de base teórico-empírica.

Para el diseño del modelo formal se realiza un abordaje teórico del tema, con base principalmente en artículos científicos vinculados a la relación entre la tasa de rendimiento de activos y la inflación. El análisis empírico está centrado en el estudio del rendimiento de las acciones en el mercado de capitales argentino en el lapso 2005-2014 y las vinculaciones con variables de interés, principalmente macroeconómicas (como la inflación, el tipo de cambio, el producto bruto interno, el rendimiento del mercado, etc.), incluyendo asimismo pruebas de control con variables microeconómicas (como el sector, el tamaño, entre otras).

\subsection{Fuentes de información y variables de interés.}

Para abordar el objetivo propuesto se construye una base de datos de panel, con registros mensuales para un período de 10 años de variables macroeconómicas y de información microeconómica de 19 empresas, constituyendo una matriz de 120 x 19. Para la elaboración de la base de datos se utiliza información secundaria recopilada por el grupo de investigación.

Los datos microeconómicos, es decir, a nivel empresa, provienen de fuentes oficiales de acceso público, como la Bolsa de Comercio de Buenos Aires (BCBA), la Comisión Nacional de Valores de la República Argentina (CNV) y el Instituto Argentino de Mercado de Capitales (IAMC). En este estudio se utiliza información de 19 de las 25 empresas que componen el Merval 25 a mayo de 2015. No fue posible completar la base de datos con el total de compañías que integran el índice por falta de información (algunas firmas no forman parte del Merval durante el período completo de relevamiento 2005-2014 o no se obtienen los Estados Contables individuales, solo los consolidados). Las 19 empresas incluidas en la base son clasificadas en tres sectores definidos ad-hoc, como se presenta a continuación:

- Sector energía y distribución: Transener SA, Endesa Costanera SA, Petróleo Brasileiro Petrobras SA, YPF SA, Edenor SA, Central Puerto, Petrobras Argentina SA, Transportadora de Gas del Sur, Petrolera Pampa SA, Transportadora de Gas del Norte SA.

- Sector industrial: Mirgor SA, Tenaris SA, Aluar SA, Molinos Río de La Plata SA.

- Sector bancario: Grupo Financiero Galicia SA, Banco Macro SA, BBVA Banco Francés SA, Banco Hipotecario SA. 
Observación: La empresa Telecom Argentina SA forma parte de la base y no fue clasificada dentro de ningún sector a fin de no adicionar una variable dummy sectorial solo por una empresa. Todas las empresas fueron analizadas individualmente, además de someterlas al análisis sectorial presentado.

Las variables microeconómicas recopiladas son:

1. El precio promedio mensual de cada acción, según su cotización de mercado obtenida de la BCBA. Con base en esta información se calcula la variable dependiente del estudio: rendimiento mensual nominal de cada empresa, en su versión logarítmica (ecuación 4) y aritmética (ecuación 5). En el análisis financiero y econométrico de series de tiempo, uno de los beneficios de emplear rendimientos, en lugar de cotizaciones, reside en la normalización ya que permite comparar activos independientemente del problema de escalas en precios (Hudson \& Gregoriou, 2010).

$$
\begin{aligned}
\widetilde{R_{l}} & =\ln \left(\frac{\text { Precio }_{t}}{\text { Precio }_{t-1}}\right) \\
\widetilde{R_{l}} & =\frac{\text { Precio }_{t}-\text { Precio }_{t-1}}{\text { Precio }_{t-1}}
\end{aligned}
$$

2. La capitalización de la empresa mensualmente, según información de la BCBA y el IAMC.

3. Información contable vinculada a cada empresa (activo, pasivo, patrimonio neto, ventas, margen bruto, resultado ordinario): se registra trimestralmente tomando la información publicada por la CNV y la BCBA. Debido a la frecuencia de recopilación de estas variables no es posible utilizarlas en diferencia para un período mensual en el análisis econométrico multivariado.

4. Los dividendos abonados a los accionistas, en efectivo y en especie, según la BCBA.

5. Sector de cada empresa, según la clasificación del IAMC.

6. Otros ratios vinculados al precio, que no serán utilizados en el presente trabajo (tales como el PER, P/VL, coeficiente beta, etc.). Las fuentes son los informes mensuales del IAMC.

Las variables macroeconómicas cuantificadas son las siguientes:

1. Rm (Merval): corresponde al rendimiento del Índice Merval en pesos ajustado por pago de dividendos y splits, obtenido de Yahoo Finance (http://finance.yahoo.com/). El índice bursátil Merval es un agregado accionario que considera empresas abiertas del mercado argentino, ponderando su participación en la cartera teórica del índice según su liquidez de acuerdo a dos criterios: número de operaciones efectuadas y volumen efectivo operado.

2. Rm (Burcap): corresponde al rendimiento del Índice Burcap, obtenido de Yahoo Finance. Se trata de un índice de mercado compuesto por las mismas acciones que el anterior, pero considera la capitalización bursátil como criterio para la ponderación relativa de cada empresa en el agregado, en lugar de la liquidez. De este modo, el Burcap refleja el comportamiento bursátil del mercado argentino considerando el tamaño de las empresas.

3. Rf1_Tbonds: rendimiento de los bonos del Tesoro de EEUU a 30 años, consultado en Yahoo Finance. 
4. Rf2_EMBI: tasa del principal indicador de riesgo país (Emerging Markets Bonds Index o Indicador de Bonos de Mercados Emergentes) correspondiente a Argentina según JP Morgan, expresada en puntos básicos ( $1 \%=100$ puntos básicos). Representa la diferencia de tasa de interés que pagan los bonos denominados en dólares, emitidos por países subdesarrollados y los Bonos del Tesoro de EEUU. Obtenido del Centro de Economía Internacional (http://www.cei.gob.ar/).

5. Rf_tbond+EMBI: tasa de rendimiento estimada mediante la suma del rendimiento de los bonos de la Tesorería de EEUU a 30 años y la tasa EMBI (Tb30y+EMBI). Los rendimientos son reexpresados en equivalente mensual.

6. Rf3_Disc: Tasa Interna de Retorno anual de los Bonos Discount en pesos con legislación argentina, descargada desde Puente Net (http://www.puentenet.com/). Se trata de títulos de renta fija emitidos por el Gobierno Nacional de la República Argentina en moneda local en el año 2003 con 30 años de vida (vencimiento en 2033). Tienen la particularidad de abonar una parte de los intereses devengados en efectivo y capitalizar la otra. Se amortizan en 20 cuotas semestrales pagaderas a partir de 2024.

7. Rf4_Boden: Tasa Interna de Retorno anual de los Boden 2012, obtenida a través de Puente Net. El Boden 2012 es un bono emitido por el Gobierno Nacional de la República Argentina en dólares estadounidenses en el año 2002, en plena crisis posconvertibilidad. El vencimiento operó en 2012. El interés pagadero semestralmente toma como referencia la tasa Libor. El bono se amortizó en 8 cuotas anuales, consecutivas y equivalentes $(12,5 \%)$ a partir de 2005 .

8. R-MervU\$S: último rendimiento mensual observado en dólares del índice Merval, descargada desde Puente Net.

9. R-BurcU\$S: último rendimiento mensual observado en dólares del índice Burcap, obtenida a través Puente Net.

10. $\operatorname{PRM(merv):~diferencia~de~rendimiento~mensual~en~dólares~calculado~tomando~como~}$ referencia de rendimiento de mercado el índice MERVAL (R-MervU\$S-Rf_tbond+EMBI).

11. PRM(burc): diferencia de rendimiento mensual calculado tomando como referencia de rendimiento de mercado el índice BURCAP (R-BurcU\$S - Rf_tbond+EMBI).

12. Inflación1_INDEC01: Índice de precios al consumidor (promedio mensual) informado por el INDEC en base enero 2001 para el período enero 2001 - diciembre 2013.

13. Inflación2_INDEC13: Índice de precios al consumidor nacional urbano (promedio mensual) informado por el INDEC en base diciembre 2013 para el período enero 2014 - diciembre 2014.

14. Inflación3_INDEC01comp: Índice de precios al consumidor (promedio mensual) en base enero 2001 para el período enero 2001 - diciembre 2014, calculado a partir de las series anteriores informadas por el INDEC (Inflación1_INDEC01 e Inflación2_INDEC13). 
15. Inflación4_INDEC04: Índice de precios al consumidor (promedio mensual) en base enero 2004 para el período enero 2001 - diciembre 2014, calculado partir de las series informadas por el INDEC (Inflación1_INDEC01 e Inflación2_INDEC13).

16. IPCCong: Índice de precios al consumidor (promedio mensual) en base abril 2011 resultante de un informe presentado por la Comisión de Libertad de Expresión de la Cámara Baja a partir de los relevamientos efectuados por diversas consultoras privadas. Comprende el periodo abril 2011 - marzo 2015.

17. IPCBevaq: Índice de precios al consumidor (promedio mensual) en base enero 2001 proveniente de un registro de precios realizado por Graciela Bevacqua (ex-directora de Precios en el INDEC). Comprende el periodo enero 2001 - marzo 2015.

18. IPCpricest: Índice de precios al consumidor (promedio mensual) en base enero 2008 creado a partir del registro de precios de la firma State Street Global Markets (ex-inflación verdadera). Comprende el periodo enero 2008 - febrero 2015.

19. CVSprivform: Coeficiente de Variación Salarial en base abril 2012 elaborado por el INDEC considerando el salario promedio por trimestre de una muestra de ocupados formales aportantes al SIJP. Comprende el periodo septiembre 2001- febrero 2015.

20. TCNof: tipo de cambio oficial pesos por dólar calculado como promedio mensual. La serie histórica se descarga del Diario Ámbito Financiero (https://www.ambito.com/contenidos/mercados.html).

21. TCNpa: tipo de cambio paralelo o no oficial pesos por dólar calculado como promedio mensual. La serie histórica se descarga del Diario Ámbito Financiero.

22. TCNcliqui: tipo de cambio pesos por dólar correspondiente a la operación conocida vulgarmente como "contado con liquidación" calculado como promedio mensual. Se conoce como contado con liquidación a la operación mediante la que una persona o empresa puede cambiar pesos argentinos por dólares en el exterior. Operan adquiriendo en Argentina títulos (bonos) de deuda pública (emitidos en dólares que cotizan en la bolsa de Nueva York) como el Boden 2012, Bonar X o Boden 2015 que luego liquidan al contado en una cuenta en el exterior (por medio de una entidad financiera o banco de confianza). La serie histórica se descarga del Diario Ámbito Financiero.

23. PIB_corriente: Producto Interno Bruto a precios de mercado en miles de pesos, obtenido de la Dirección Nacional de Cuentas Nacionales del INDEC.

24. PIB_cte 04: Producto Interno Bruto en miles de pesos constantes con base 2004, obtenido de la Dirección Nacional de Cuentas Nacionales del INDEC. La frecuencia trimestral de las variables PIB provoca que no sea consistente utilizarlas en diferencia para un período mensual en el análisis econométrico multivariado.

Sistematizando la información anteriormente descripta, se consigue armar una base de datos de panel, donde la variable tiempo (t) es mensual, presentando datos para 120 meses desde 2005 a 2014, y la variable de panel (n) representa cada empresa. La base presenta un panel de $19 \times 120$ fuertemente balanceado, alcanzando un número de 2.280 observaciones. La variable dependiente es 
el rendimiento requerido del conjunto de acciones en el mercado de capitales argentinos. Las variables independientes se presentan en la Tabla A.1 incluida en el Anexo.

\subsection{Métodos de análisis.}

Para llevar a cabo el estudio se utilizan métodos estadísticos y econométricos (válidos para un panel de datos) en pos de analizar la información obtenida. Los datos se procesan en una planilla de cálculo y son exportados como archivo de extensión $d t a$ al software econométrico Stata SE versión 11.1. Inicialmente se realizan análisis univariados y bivariados, estudiando las tendencias de las variables relevadas y examinando la correlación entre las mismas, respectivamente. Luego, a partir de la información brindada por estas pruebas, se efectúan estimaciones multivariadas con diferentes especificaciones.

Previo a los análisis en sí mismos, se realizan una serie de pruebas estadísticas a fin de verificar las propiedades de los datos, empleando para ello el mencionado software econométrico. En primer lugar, se examina la estacionariedad de las series. Este procedimiento resulta necesario cuando se analizan series temporales ya que permite identificar la potencial presencia de algún tipo de tendencia en las series bajo estudio. Si éste fuera el caso, existe la posibilidad de arribar a conclusiones erróneas, incurriendo en lo que se denomina una regresión espuria, esto es, una regresión que muestra la existencia de una relación estadísticamente significativa entre las variables cuando, en realidad, el resultado sólo es consecuencia del movimiento tendencial de las series (Gujarati \& Porter, 2011). Para el análisis de la estacionariedad de las series se aplicaron distintas pruebas de raíz unitaria: DickeyFuller (DF), Augmented Dickey-Fuller (ADF), Phillips-Perron (PP) y Zivot Andrews (ZA). Éste último test permite comprobar si se trata de una serie estacionaria con quiebre en la tendencia, en el intercepto o en ambos.

Al trabajar con series de tiempo pueden encontrarse diferentes situaciones (Montero, 2013):

a) Si las variables son estacionarias en niveles (es decir, integradas de orden 0 ) los coeficientes estimados por MCO muestran relaciones de largo plazo.

b) Si las variables no son estacionarias en niveles, pero son integradas del mismo orden (por ejemplo I(1): estacionarias en primera diferencia) y están cointegradas se puede realizar la regresión por MCO para estimar los efectos a largo plazo y el modelo de corrección de errores para obtener los efectos a corto plazo.

c) Si las series son no estacionarias del mismo orden y no están cointegradas: no puede estimarse la relación entre ambas porque la regresión es espuria.

d) Si las series son no estacionarias de orden distinto entre sí: no puede estimarse la relación entre ambas.

El presente estudio encuadra en el supuesto b, por lo tanto, además de analizar la estacionariedad de las series se evaluó su cointegración. Por definición, las variables temporales $x t$ e $y t$ estacionarias de orden 1 (es decir son I(1)) están cointegradas cuando puede practicarse una regresión lineal $y t=a+b x t+u t$, y el residuo es estacionario, es decir, $u t=-a+y t+b x t$ es $\mathrm{I}(0)$. Entonces, los requisitos para definir la cointegración son: (i) que dos variables sean estacionarias de orden 1 y (ii) que exista una combinación lineal de ambas que sea estacionaria de orden 0 . Cuando ambas condiciones se cumplen se dice que las variables están cointegradas. Cointegración significa que existe una relación, a largo plazo, entre las variables. En definitiva, si $x t$ e $y t$ están cointegradas significa que, aunque crezcan en el tiempo $(t)$, lo hacen de una forma completamente acompasada, de forma que el error entre ambas no crece. Es decir, si en la regresión $y=a+b x+u, u$ es estacionario 
(I(0)) entonces $b$ refleja relaciones de largo plazo. Para testar la cointegración se aplica el método de Engle y Granger que consiste en estimar los residuos del modelo de regresión y aplicar pruebas de estacionariedad a los residuos estimados.

Adicionalmente a las pruebas de estacionariedad y cointegración realizadas sobre las series de tiempo, se efectúan diferentes pruebas estadísticas sobre las regresiones elegidas, de modo de poder verificar el cumplimiento de los supuestos básicos del método de mínimos cuadrados ordinarios.

El análisis multivariado se realiza utilizando alternativamente los siguientes modelos para datos de panel (Wooldridge, 2002, 2015; Greene, 2003; Gujarati, 2004; Kennedy, 2008):

- Regresión agrupada o pool: Se utiliza el método de mínimos cuadrados ordinario, omitiendo las dimensiones de espacio y tiempo, es decir, fusionando el panel. Se realiza el Test de Breusch y Pagan para verificar si existe heteroscedasticidad. Para ajustar la regresión por heteroscedasticidad se utiliza el método de White.

- Modelo de efectos aleatorios o random effects: Esta regresión implica suponer que el intercepto no es el mismo para todas las unidades transversales. Se estima el modelo y se testea si efectivamente existen efectos no observables. Para ello se utiliza la Prueba del Multiplicador de Lagrange (LM) de Breusch y Pagan para efectos aleatorios. El test de Hausman permite analizar si en caso de existir efectos inobservables deben modelarse bajo efectos fijos o aleatorios.

- Modelo de efectos fijos o fixed effects: Supone que las diferencias entre unidades no son aleatorias, sino constantes o fijas. Para probar si es adecuada la estimación con efectos fijos se crean variables dicotómicas de intersección diferencial. Se realiza una prueba de F restrictiva para analizar si corresponde incluir estas variables dicotómicas de estado.

- Modelo de efectos temporales o two-way fixed effects: Agrega variables dicotómicas que capturan eventos comunes a todos los estados para determinados momentos de tiempo (por ejemplo, años de crisis).

\section{Resultados.}

\subsection{Análisis descriptivo de tendencias.}

Se realizan análisis de estacionariedad de las series encontrándose los resultados presentados en la Tabla A.2 del Anexo. En líneas generales, se observa que todas las series son integradas de primer orden, esto es, estacionarias en primeras diferencias. Para variables como la inflación y el tipo de cambio, esto implica efectuar las pruebas con los diferenciales de cada serie, generando variables a tal efecto. Por ejemplo, para la inflación el diferencial se define como:

$$
\text { d.inflación }=\Delta \text { inflación }=\text { inflación }{ }_{t}-\text { inflación }_{\mathrm{t}-1}
$$

Por el contrario, en los casos de métricas de rendimientos, siendo que su cálculo ya implica estimar una diferencia o variación entre períodos, la estacionariedad se encuentra sobre las variables sin ajustar. Por su naturaleza, el rendimiento se estima como:

$$
\left(\text { variable }_{t}-\text { variable }_{t-1}\right) / \text { variable }_{t-1} ; \text { o bien, } \ln \left(\text { variable }_{t} / \text { variable }_{t-1}\right)
$$


La base cuenta con 2078 datos de rendimientos de acciones con media en 1,14\% mensual (existen algunos datos perdidos para dos de las empresas) y la inflación presenta 2.280 datos inflacionarios, con una media en niveles de 176,53. En la Ilustración 1 se presentan las series de rendimientos de cada empresa del panel (de 1 a 19) y la serie de diferencial inflacionario sobre la variable Inflación4_INDEC04, para analizar las tendencias gráficamente. En términos agregados, puede observarse que los rendimientos siguen en promedio movimientos sin tendencia definida, mientras que la serie inflacionaria presenta un diferencial con tendencia creciente y cada vez con mayor amplitud de movimientos.

Ilustración 1. Series temporales de rendimiento de cada empresa y diferenciales inflacionarios.
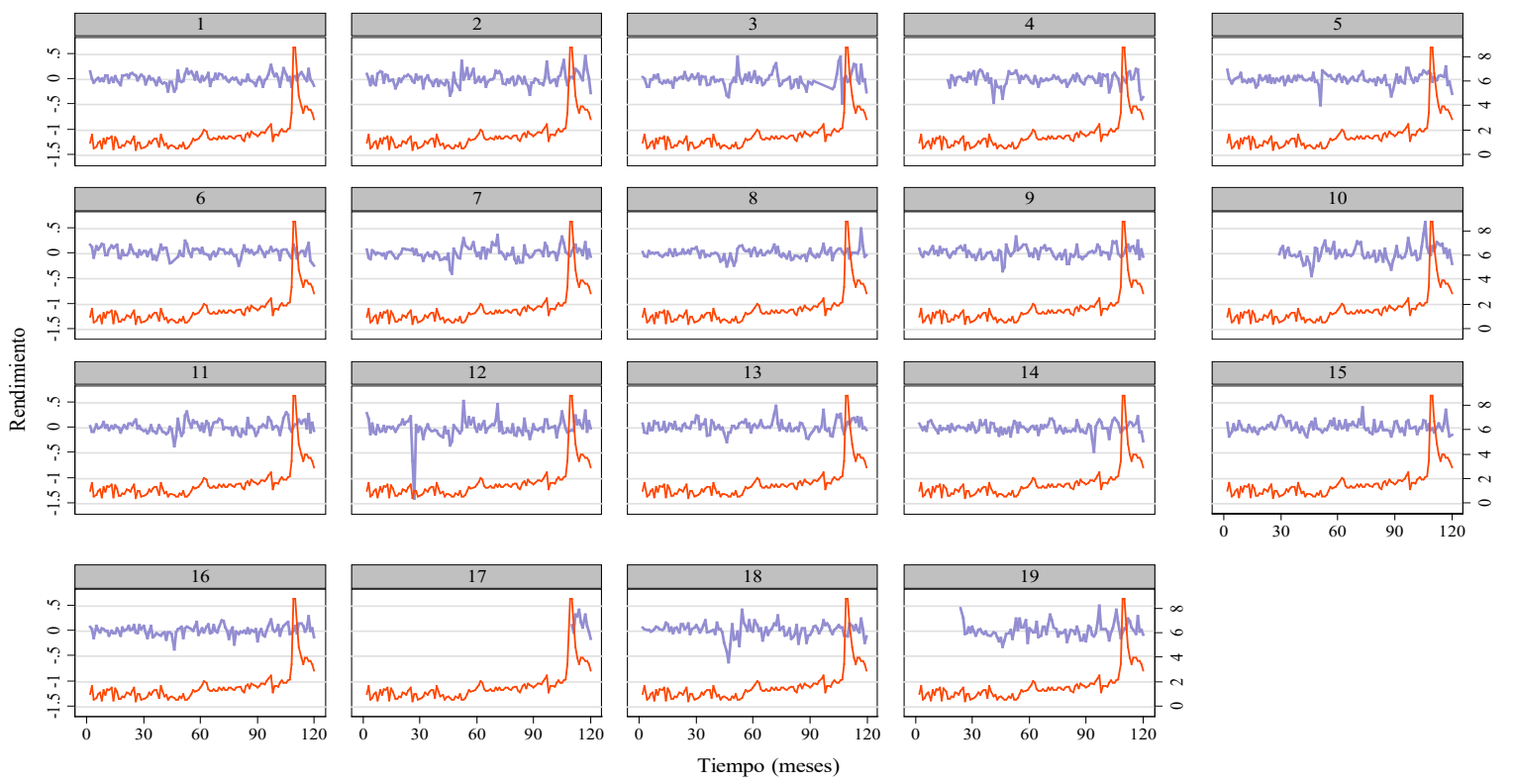

Rendimiento activo

Inflación

Fuente: Elaboración propia a partir de software econométrico.

Se examinan asimismo los coeficientes de correlación entre el rendimiento de las acciones y las diferentes medidas de inflación, en niveles y en diferencias (Tabla 2), siendo todos ellos positivos. Debemos aclarar que el coeficiente de correlación entre todas las variables que monitorean la inflación en niveles es de 0,99; mientras que en diferencias arroja valores entre 0,21 y 0,93.

Tabla 2. Correlaciones entre el rendimiento y las variables inflacionarias $\left(\pi_{t}\right)$.

\begin{tabular}{|c|c|c|c|c|c|}
\hline$\pi_{\boldsymbol{t}}$ en niveles & $\pi_{t}$ INDEC & $\pi_{t}$ Cong & $\pi_{t}$ Bevaq. & $\pi_{t}$ Pricestat & $\pi_{t}$ CVS \\
\hline Rendimiento $\left(R_{i t}\right)$ & 0,2364 & 0,2378 & 0,2416 & 0,2382 & 0,2508 \\
\hline$\pi_{\boldsymbol{t}}$ en diferencias & $\pi_{t}$ INDEC & $\pi_{t}$ Cong & $\pi_{t}$ Bevaq. & $\pi_{t}$ Pricestat & $\pi_{t}$ CVS \\
\hline Rendimiento $\left(R_{i t}\right)$ & 0,1540 & 0,2086 & 0,2359 & 0,0919 & 0,1607 \\
\hline
\end{tabular}

Fuente: Elaboración propia. 
En cuanto a las series temporales de rendimiento de los índices de mercado (Ilustración 2), las variables presentan una conducta correlacionada positivamente, siguiendo en promedio el mismo patrón de comportamiento. Por el contrario, la tasa libre de riesgo ajustada por riesgo país (T-bonds + EMBI) presenta una conducta más estable, es decir, de menor varianza, lo que resulta esperable por su naturaleza.

Ilustración 2. Series temporales de rendimientos de índices de mercado y títulos públicos.

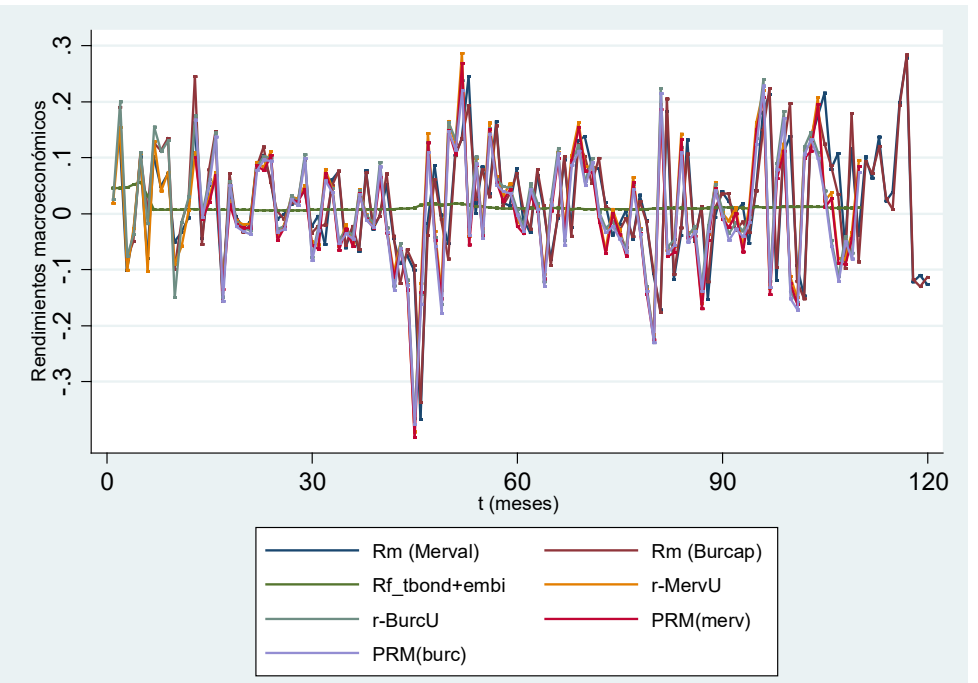

Fuente: Elaboración propia a partir de software econométrico.

En particular, se analiza la correlación entre los rendimientos de los activos versus los de diferentes títulos libres de riesgo, presentándose correlaciones positivas con tasas libres de riesgo internacionales, como la de los bonos del tesoro de EEUU, mientras que la correlación es negativa cuando se utilizan rendimientos libres de riesgo que incorporan el riesgo país, como el rendimiento que surge de la tasa T-bonds+EMBI, de los bonos Discount o de los bonos Boden (Tabla 3).

Tabla 3. Correlaciones entre el rendimiento y tasas libre de riesgo $\left(R f_{t}\right)$.

\begin{tabular}{|c|c|c|c|c|}
\hline $\boldsymbol{R} \boldsymbol{f}_{\boldsymbol{t}}$ & $R f_{t}$ T-bonds & $R f_{t}$ T-b+EMBI & $R f_{t}$ Disc. & $R f_{t}$ Boden \\
\hline Rendimiento $\left(R_{i t}\right)$ & 0,1663 & $-0,3163$ & $-0,2429$ & $-0,1988$ \\
\hline
\end{tabular}

Fuente: Elaboración propia.

Si se estiman las correlaciones entre los títulos, se encuentra que el rendimiento de los T-bonds presenta una correlación negativa entre $-0,91$ y $-0,83$ con cualquiera de los otros proxies de la tasa libre de riesgo, que tienen un plus por incorporar el riesgo país. Por el contrario, entre las tasas libre de riesgo que contemplan el riesgo país, la correlación es positiva y alta, de entre 0,93 y 0,94.

Luego se realiza un análisis similar para conocer los coeficientes de correlación entre el rendimiento de las acciones y las diferentes medidas de rendimiento de mercado (Tabla 4), siendo la 
correlación significativamente más fuerte cuando se utilizan los rendimientos de los índices bursátiles Merval y Burcap en moneda local (\$).

Tabla 4. Correlaciones entre el rendimiento y tasas de rendimiento de mercado $\left(R m_{t}\right)$.

\begin{tabular}{|c|c|c|c|c|c|c|}
\hline $\boldsymbol{R m}_{\boldsymbol{t}}$ & $\begin{array}{c}R m_{t} \text { Merval } \\
\$\end{array}$ & $\begin{array}{c}R m_{t} \text { Burcap } \\
\$\end{array}$ & $\begin{array}{c}R m_{t} \text { Merval } \\
\text { US\$ }\end{array}$ & $\begin{array}{c}R m_{t} \text { Burcap } \\
\text { US\$ }\end{array}$ & $\begin{array}{c}\text { PR }\left(R m_{t}\right) \\
\text { Merval US\$ }\end{array}$ & $\begin{array}{c}\text { PR }\left(R m_{t}\right) \\
\text { Burcap US\$ }\end{array}$ \\
\hline Rendimiento $\left(R_{i t}\right)$ & 0,4692 & 0,4278 & 0,0961 & 0,0787 & 0,0987 & 0,0811 \\
\hline
\end{tabular}

Fuente: Elaboración propia.

Si se estudian las correlaciones cruzadas entre los proxies de $R m_{t}$, los índices Merval y Burcap en la misma moneda, sea pesos o dólares, presentan una correlación positiva y alta de 0,96 ó 0,97 . Si se calculan las correlaciones entre los índices en diferentes monedas, los coeficientes bajan significativamente, rondando un valor de 0,25 . Y, naturalmente, el premio del riesgo de cada índice con el índice en cuestión presenta una relación positiva casi perfecta de 0,999.

En relación al tipo de cambio, la tendencia es creciente sobre todo en la segunda mitad del período bajo estudio (Ilustración 3), en donde se observa un incremento significativo en la varianza, principalmente del tipo de cambio paralelo (Ilustración 4).

Ilustración 3.

Series temporales del tipo de cambio (US\$ $\mathbf{\$})$.

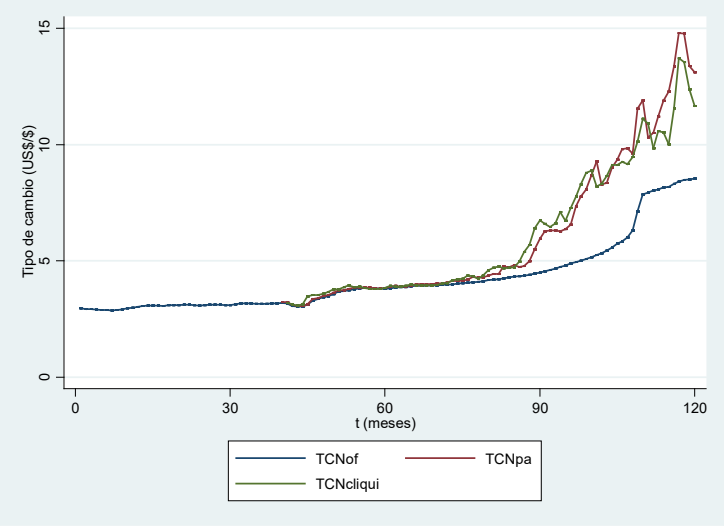

Fuente: Elaboración propia a partir de software econométrico.
Ilustración 4.

Diferenciales temporales del tipo de cambio.

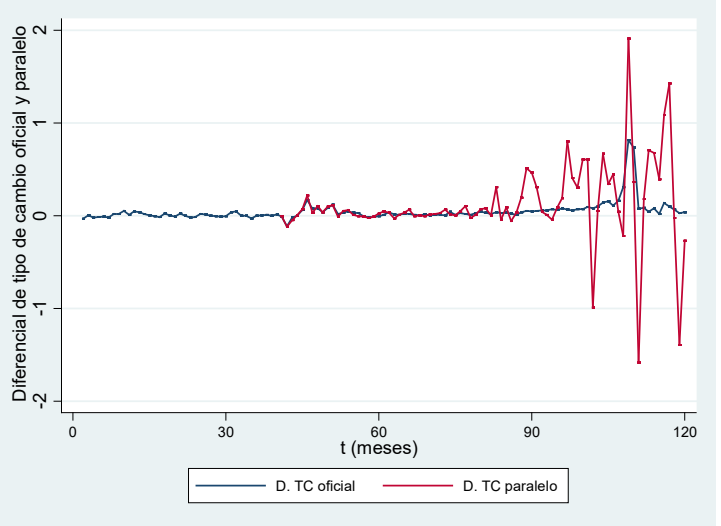

Fuente: Elaboración propia a partir de software econométrico.

Cuando se estudian las correlaciones de los rendimientos de los activos y el tipo de cambio en niveles se encuentran coeficientes positivos; mientras que, si se trabaja con el tipo de cambio en diferencias, el contado con liquidación pierde relevancia y el tipo de cambio oficial presenta una correlación negativa (Tabla 5).

Al analizar la correlación en niveles entre el tipo de cambio oficial y el paralelo se encuentra un valor alto, de 0,965 , al igual que cuando se correlaciona el tipo de cambio oficial con el contado con liquidación, donde el coeficiente es de 0,946 . La correlación entre el tipo de cambio paralelo con el contado con liquidación es de 0,986 . 
Si se estudian las correlaciones en diferencias, los coeficientes son algo menores: el tipo de cambio oficial y el paralelo se correlacionan en 0,58 ; el tipo de cambio oficial y el contado con liquidación en 0,53 ; y, por último, el tipo de cambio paralelo y el contado con liquidación en 0,36 .

Tabla 5. Correlaciones entre el rendimiento y las variables de tipo de cambio $\left(\operatorname{TC}_{t}\right)$.

\begin{tabular}{|c|c|c|c|}
\hline $\boldsymbol{T C}_{\boldsymbol{t}}$ en niveles & $T C_{t}$ oficial & $T C_{t}$ paralelo & $T C_{t}$ c. liquid \\
\hline Rendimiento $\left(R_{i t}\right)$ & 0,1621 & 0,1590 & 0,1376 \\
\hline $\boldsymbol{T} \boldsymbol{C}_{\boldsymbol{t}}$ en diferencias & $T C_{t}$ oficial & $T C_{t}$ paralelo & $T C_{t}$ c. liquid \\
\hline Rendimiento $\left(R_{i t}\right)$ & $-0,0018$ & 0,1691 & 0,0002 \\
\hline
\end{tabular}

Fuente: Elaboración propia.

En la Tabla 6 se presenta la correlación entre los rendimientos de los activos y el PIB en términos corrientes y constantes (base 2004), encontrándose resultados para los coeficientes de correlación menores a 0,10 , valores bajos posiblemente vinculados a la diferencia de frecuencia de ambas series. La variable PIB no se puede calcular en diferencias con periodicidad mensual por lo explicado anteriormente. Entre las series de PIB se presenta una correlación positiva y alta, con un coeficiente de 0,894 .

Tabla 6. Correlaciones entre el rendimiento y producto bruto interno $\left(P I B_{t}\right)$.

\begin{tabular}{|c|c|c|}
\hline $\boldsymbol{P I B}_{\boldsymbol{t}}$ en niveles & $P I B_{t}$ corriente & $P I B_{t}$ constante \\
\hline Rendimiento $\left(R_{i t}\right)$ & 0,0982 & 0,0343 \\
\hline
\end{tabular}

Fuente: Elaboración propia.

Por último, en cuanto a las variables de tamaño de la empresa en vinculación a los rendimientos, se presentan los resultados en la Tabla 7. Nuevamente las variables de tamaño medidas contablemente no se pueden calcular en diferencias con periodicidad mensual. Entre las variables de tamaño, activo y patrimonio neto se correlacionan en 0,88 , mientras que la capitalización presenta una correlación con las medidas contables de tamaño que ronda entre 0,74 y 0,86 respectivamente.

Estos análisis de tendencias y correlaciones permiten predecir con qué variables explicativas se espera una relación más fuerte, cuáles variables no pueden ser incluidas en simultáneo por la potencial multicolinealidad y cuáles serían las especificaciones posibles dadas las limitaciones en los datos. Este análisis se desarrolla en la siguiente subsección.

Tabla 7. Correlaciones entre el rendimiento y tamaño $\left(T_{i t}\right)$.

\begin{tabular}{|c|c|c|c|}
\hline $\boldsymbol{T}_{\boldsymbol{i t}}$ en niveles & $T_{i t}$ según activos & $T_{i t}$ según patrimonio neto & $T_{i t}$ según capitalización \\
\hline Rendimiento $\left(R_{i t}\right)$ & 0,0864 & 0,0585 & 0,1125 \\
\hline
\end{tabular}

Fuente: Elaboración propia. 


\subsection{Análisis econométrico.}

El modelo a estimar bajo diferentes especificaciones es el presentado en la ecuación 4, donde el rendimiento observado de las acciones se encuentra linealmente relacionado con variables macroeconómicas, como la inflación, el tipo de cambio, la tasa de rendimiento libre de riesgo, el rendimiento de mercado, así como con variables dicotómicas de efectos temporales para los años 2008, 2011 y 2013, y con variables de control binarias que identifican la pertenencia a diferentes sectores de la economía y efectos fijos por empresas.

Las variables dummies temporales se definen por factores político-institucionales que condicionan el contexto económico, de los que se espera influyan en el desempeño de las empresas: el año 2008 por la crisis financiera internacional, el año 2011 por la implementación de la política de regulación del mercado cambiario argentino (percepción y restricciones para compra de divisas) y el año 2013 debido al aumento de la percepción sobre la compra de divisas y ampliación de operaciones alcanzadas.

$$
\begin{gathered}
\widetilde{R}_{\iota}=\alpha+\beta_{1} * \pi_{t}+\beta_{2} * T C_{t}+\beta_{3} * R f_{t}+\beta_{4} * R m_{t}+\beta_{5} * R m^{\prime}{ }_{t}+\beta_{6} a 2008+ \\
\beta_{7} a 2011+\beta_{8} a 2013+\beta_{9} s .1+\beta_{10} s .2+\beta_{i} e_{i}+\tilde{\varepsilon}_{i}
\end{gathered}
$$

Debemos aclarar que el modelo no incluye la variable PIB debido a que la misma no es estacionaria en niveles, no siendo consistente trabajar con sus diferencias por la periodicidad trimestral del dato. Asimismo, la falta de inclusión de una variable de control por tamaño de las firmas se debe a la presencia de endogeneidad (si se usase la capitalización bursátil) y el problema de la no estacionariedad en niveles, en caso de emplearse las variables de información contable de periodicidad trimestral.

Se prueban alternativamente varios modelos con diferentes especificaciones siguiendo la ecuación 6, informándose los resultados para cinco de ellos que fueron seleccionados en la Tabla 8 , a partir de los cuales se desprenden los principales hallazgos. Aparecen en esta tabla los coeficientes estimados para las variables independientes y, entre paréntesis, los errores estándar de los mismos.

\begin{tabular}{|c|c|c|c|c|c|}
\hline Variables / Modelos & (I) & (II) & (III) & (IV) & (V) \\
\hline Inflación INDEC base 2004 & $\begin{array}{c}0,0179 \\
(0,0049) \\
* * *\end{array}$ & $\begin{array}{c}0,0144 \\
(0,0051) \\
* * *\end{array}$ & $\begin{array}{c}-0,0089 \\
(0,0028) \\
* * *\end{array}$ & & \\
\hline Inflación PriceStat • & & & & $\begin{array}{c}-0,0045 \\
(0,0018) \\
* * *\end{array}$ & $\begin{array}{c}-0,0041 \\
(0,0012) \\
* * *\end{array}$ \\
\hline Tipo de cambio oficial & $\begin{array}{c}-0,1867 \\
(0,0500) \\
* * *\end{array}$ & $\begin{array}{c}-0,1783 \\
(0,0515) \\
* * *\end{array}$ & & $\begin{array}{c}0,0475 \\
(0,0357)\end{array}$ & \\
\hline Tipo de cambio paralelo & & & $\begin{array}{c}0,0383 \\
(0,0115) \\
* * *\end{array}$ & & $\begin{array}{c}0,0312 \\
(0,0104) \\
* * *\end{array}$ \\
\hline Rendimiento t-bonds & $\begin{array}{c}0,0181 \\
(0,0037) \\
* * *\end{array}$ & & & & \\
\hline Rendimiento t-bonds + EMBI & & $\begin{array}{c}-0,5838 \\
(0,3105) \\
* *\end{array}$ & $\begin{array}{c}-8,1453 \\
(1,2752) \\
* * *\end{array}$ & $\begin{array}{c}-8,6944 \\
(1,3249) \\
* * *\end{array}$ & $\begin{array}{c}-8,2456 \\
(1,2782) \\
* * *\end{array}$ \\
\hline
\end{tabular}

Tabla 8. Modelos de regresión del rendimiento de acciones en el mercado argentino. 


\begin{tabular}{|c|c|c|c|c|c|}
\hline Variables / Modelos & (I) & (II) & (III) & (IV) & (V) \\
\hline Rendimiento Merval \$ & $\begin{array}{c}0,5300 \\
(0,0280) \\
* * *\end{array}$ & & $\begin{array}{c}0,5299 \\
(0,0315) \\
* * *\end{array}$ & $\begin{array}{c}0,5461 \\
(0,0301) \\
* * *\end{array}$ & $\begin{array}{c}0,5252 \\
(0,0317) \\
* * *\end{array}$ \\
\hline Rendimiento Burcap \$ & & $\begin{array}{c}0,4692 \\
(0,0280) \\
* * *\end{array}$ & & & \\
\hline Rendimiento Merval US\$ & $\begin{array}{c}-0,0519 \\
(0,0253) \\
* *\end{array}$ & & & & \\
\hline Rendimiento Burcap US\$ & & $\begin{array}{c}-0,0800 \\
(0,0256) \\
* * *\end{array}$ & & & \\
\hline Año 2008 & $\begin{array}{c}-0,0478 \\
(0,0082) \\
* * *\end{array}$ & $\begin{array}{c}-0,0540 \\
(0,0084) \\
* * *\end{array}$ & $\begin{array}{c}-0,0620 \\
(0,0106) \\
* * *\end{array}$ & $\begin{array}{c}-0,0561 \\
(0,0091) \\
* * *\end{array}$ & $\begin{array}{c}-0,0606 \\
(0,0105) \\
* * *\end{array}$ \\
\hline Año 2011 & $\begin{array}{c}-0,0211 \\
(0,0079) \\
* * *\end{array}$ & $\begin{array}{c}-0,0328 \\
(0,0080) \\
* * *\end{array}$ & $\begin{array}{c}-0,0374 \\
(0,0089) \\
* * *\end{array}$ & $\begin{array}{c}-0,0357 \\
(0,0087) \\
* * *\end{array}$ & $\begin{array}{c}-0,0360 \\
(0,0088) \\
* * *\end{array}$ \\
\hline Año 2013 & $\begin{array}{c}0,0340 \\
(0,0083) \\
* * *\end{array}$ & $\begin{array}{c}0,0301 \\
(0,0081) \\
* * *\end{array}$ & $\begin{array}{c}0,0224 \\
(0,0083) \\
* * *\end{array}$ & $\begin{array}{c}0,0263 \\
(0,0083) \\
* * *\end{array}$ & $\begin{array}{c}0,0246 \\
(0,0084) \\
* * *\end{array}$ \\
\hline Sector bancario & $\begin{array}{c}-0,0091 \\
(0,0066)\end{array}$ & $\begin{array}{c}-0,0091 \\
(0,0067)\end{array}$ & $\begin{array}{c}0,0057 \\
(0,0083)\end{array}$ & & $\begin{array}{c}0,0057 \\
(0,0083)\end{array}$ \\
\hline Sector energía y transporte & $\begin{array}{c}-0,0106 \\
(0,0055) \\
* *\end{array}$ & $\begin{array}{c}-0,0107 \\
(0,057) \\
* *\end{array}$ & $\begin{array}{c}-0,0071 \\
(0,0070)\end{array}$ & $\begin{array}{c}-0,0124 \\
(0,0067) \\
*\end{array}$ & $\begin{array}{c}-0,0070 \\
(0,0070)\end{array}$ \\
\hline Sector industrial & & & & $\begin{array}{c}-0,0057 \\
(0,0081)\end{array}$ & \\
\hline Constante & $\begin{array}{c}-0,0811 \\
(0,0178) \\
* * *\end{array}$ & $\begin{array}{c}0,0067 \\
(0,0083)\end{array}$ & $\begin{array}{c}0,1049 \\
(0,0167) \\
* * *\end{array}$ & $\begin{array}{c}0,1174 \\
(0,0181) \\
* * *\end{array}$ & $\begin{array}{c}0,1061 \\
(0,0167) \\
* * *\end{array}$ \\
\hline
\end{tabular}

Fuente: Elaboración propia.

En la Tabla 9 se presentan los resultados de diferentes pruebas estadísticas efectuadas sobre los modelos elegidos, a fin de verificar el cumplimiento de los supuestos básicos del método de mínimos cuadrados ordinarios y la condición de cointegración. Asimismo, se estiman estadísticos que representan la bondad de ajuste de los modelos, situada entre el $22 \%$ y el $32 \%$, siendo aceptables dichos niveles para el análisis con datos de panel. Todos modelos presentan significatividad conjunta según el test $\mathrm{F}$.

En cuanto a los supuestos básicos para la estimación mediante MCO se determina la no existencia de autocorrelación en los residuos, a partir del análisis gráfico (Ilustraciones A.1-A.5 del Anexo). En relación a la homoscedasticidad del error, en los modelos I y II no se rechaza la hipótesis nula de varianza contante, por lo que es homoscedástico. En los modelos III, IV y V, el test de White no permite abordar a la misma conclusión. A pesar de ello, debido al tamaño de la muestra, puede afirmarse que los coeficientes estimados en las regresiones son consistentes y convergen a su valor verdadero. En referencia a la normalidad, si bien según el test de Jarque-Bera midiendo los atributos 
de asimetría y curtosis, se rechaza la hipótesis nula en los cinco modelos, el teorema de Gauss-Markov demuestra que de todos modos los estimadores de mínimos cuadrados ordinarios son los mejores lineales insesgados (Gujarati \& Porter, 2011).

Tabla 9. Pruebas de propiedades de las regresiones seleccionadas.

\begin{tabular}{|c|c|c|c|c|c|}
\hline Variables / Modelos & (I) & (II) & (III) & (IV) & (V) \\
\hline $\begin{array}{l}\mathrm{R}^{2} \text { ajustado } \\
\text { (bondad de ajuste) }\end{array}$ & 0,2542 & 0,2190 & 0,3221 & 0,3141 & 0,3227 \\
\hline $\begin{array}{l}\text { Test F } \\
\text { (significatividad conjunta) }\end{array}$ & $\begin{array}{l}\text { F: } 65,31 \\
\text { p: } 0,0000\end{array}$ & $\begin{array}{l}\text { F: } 53,91 \\
\text { p: } 0,0000\end{array}$ & $\begin{array}{l}\text { F: } 66,93 \\
\text { p: } 0,0000\end{array}$ & $\begin{array}{l}\text { F: } 67,31 \\
\text { p: } 0,0000\end{array}$ & $\begin{array}{c}\text { F: } 67,11 \\
\text { p: } 0,0000\end{array}$ \\
\hline $\begin{array}{l}\text { Test White } \\
\text { (homoscedasticidad) }\end{array}$ & $\begin{array}{l}\chi^{2}: 39,1265 \\
\text { p: } 0,9578\end{array}$ & $\begin{array}{c}\chi^{2}: 38,8818 \\
\text { p: } 0,9604\end{array}$ & $\begin{array}{c}\chi^{2}: 129,033 \\
\text { p: } 4,8 \mathrm{e}-10\end{array}$ & $\begin{array}{c}\chi^{2}: 134,568 \\
\text { p: } 7,3 \mathrm{e}-11\end{array}$ & $\begin{array}{c}\chi^{2}: 126,7296 \\
\text { p: } 1,0 \mathrm{e}-09\end{array}$ \\
\hline $\begin{array}{l}\text { Test de Jarque-Bera } \\
\text { (normalidad) }\end{array}$ & $\begin{array}{l}\text { S: } 0,0000 \\
\text { K: } 0,0000\end{array}$ & $\begin{array}{l}\text { S: } 0,0000 \\
\text { K: } 0,0000\end{array}$ & $\begin{array}{l}\text { S: } 0,0000 \\
\text { K: } 0,0000\end{array}$ & $\begin{array}{l}\text { S: } 0,0000 \\
\text { K: } 0,0000\end{array}$ & $\begin{array}{l}\text { S: } 0,0000 \\
\text { K: } 0,0000\end{array}$ \\
\hline $\begin{array}{l}\text { Test de Engle-Granger } \\
\text { (cointegración) }\end{array}$ & $\begin{array}{l}\beta_{l . r}:-0,8797 \\
\text { p: } 0,0000\end{array}$ & $\begin{array}{l}\beta_{l . r}:-0,8355 \\
\text { p: } 0,0000\end{array}$ & $\begin{array}{l}\beta_{l . r}:-0,9362 \\
\text { p: } 0,0000\end{array}$ & $\begin{array}{l}\beta_{l . r}:-0,9576 \\
\text { p: } 0,0000\end{array}$ & $\begin{array}{l}\beta_{l . r}:-0,9527 \\
\text { p: } 0,0000\end{array}$ \\
\hline $\begin{array}{l}\text { Método de corrección de } \\
\text { errores }\end{array}$ & $\begin{array}{l}\beta_{l . r}:-0,8460 \\
\text { p: } 0,0000\end{array}$ & $\begin{array}{l}\beta_{l . r}:-0,8165 \\
\text { p: } 0,0000\end{array}$ & $\begin{array}{l}\beta_{l . r}:-0,8821 \\
\text { p: } 0,0000\end{array}$ & $\begin{array}{l}\beta_{l . r}:-0,8972 \\
\text { p: } 0,0000\end{array}$ & $\begin{array}{l}\beta_{l . r}:-0,8899 \\
\text { p: } 0,0000\end{array}$ \\
\hline
\end{tabular}

Fuente: Elaboración propia.

Al evaluar la cointegración, con el método de Engle-Granger, el residuo de la combinación lineal de las variables en primera diferencia resulta estacionario, por lo que se puede afirmar que las variables están cointegradas. Esto es, exhiben una relación de largo plazo y, por ende, los coeficientes pueden considerarse estables y consolidados.

En relación a los modelos seleccionados, los resultados más interesantes y controversiales se presentan en torno a las variables inflación y tipo de cambio, dado que los efectos encontrados son los opuestos si se comparan los modelos I y II, donde las variables se monitorean con estadísticas oficiales; versus las regresiones III, IV y V, donde una o las dos variables están medidas por indicadores alternativos. Si bien por su naturaleza estas variables están fuertemente correlacionadas y podrían provocar un problema de multicolinealidad, siendo que en este caso se trabaja con series intervenidas (y en diferencias), se pueden utilizan ambas variables simultáneamente en las regresiones pues la correlación entre ellas nunca supera el $75 \%$.

En particular, con las estadísticas oficiales, la inflación presenta un efecto positivo cuando el del tipo del cambio es negativo (Tabla 10). El efecto positivo de la inflación, considerando que los rendimientos de los activos están medidos en términos nominales, brinda soporte del efecto de Fisher y es consistente con los hallazgos de Choudhry (2001). El efecto negativo del tipo de cambio puede interpretarse como la disminución en las operaciones de la firma, que derivan en un menor rendimiento.

Cuando se utilizan alternativas de medición para las variables inflación (PriceStat) y tipo de cambio (paralelo) los resultados se revierten (Tabla 10). De hecho, estos podrían considerarse más confiables debido a que la correlación entre las explicativas es sustancialmente menor y las mismas capturan expectativas del mercado, no reflejadas en las series oficiales por no ser producto de la interacción entre la oferta y la demanda. Este fenómeno se continuará estudiando para poder concluir acerca del "verdadero" efecto de la inflación y el valor de la divisa sobre los rendimientos de acciones en el mercado de capitales argentino. 
Tabla 10. Efectos encontrados para inflación y tipo de cambio sobre el rendimiento de los activos.

\begin{tabular}{|l|c|r|r|r|}
\hline & Modelos I y II & Modelos III & Modelos IV & Modelos V \\
\hline Inflación en diferencias & + & \multicolumn{3}{|c|}{-} \\
\hline Tipo de cambio en diferencias & - & \multicolumn{3}{|c|}{+} \\
\hline $\begin{array}{l}\text { Correlación entre las variables } \\
\text { explicativas }\end{array}$ & 0,747 & 0,219 & 0,552 & 0,035 \\
\hline
\end{tabular}

Fuente: Elaboración propia.

Los hallazgos referidos a la relación positiva entre rendimiento accionario e inflación (medida a partir de estadísticas oficiales) son consistentes con las estimaciones multivariadas de Choudhry (2001) para el periodo 1981-1998 y con las correlaciones de Dapena (2013) y Lanche-Bonde (2015) para el lapso 2003-2013. Por su parte, el vínculo negativo encontrado en el presente estudio entre rendimientos nominales e inflación medida con fuentes alternativas no puede contrastarse con ningún antecedente, por ser el primero que emplea datos no oficiales para aproximar la variación en el nivel de precio. Sin embargo, pueden mencionarse tres relaciones negativas entre inflación oficial y rendimientos accionarios verificadas en el contexto argentino: con retornos reales (Choudhry, 2001 [período 1981 y 1998]; López, 2016 [lapso 2002-2014]) y nominales (Guerra, 2012 [brecha 20022013]).

Asimismo, se destacan algunos estudios que no han encontrado un vínculo estadísticamente significativo entre los precios de las especies y la inflación en Argentina como Raele (2011) y Guerra (2012) para el periodo de convertibilidad (1993-2001). En lo que respecta al tipo de cambio, el trabajo de Raele (2011) es el único antecedente identificado para contrastar, cuyos hallazgos muestran una relación positiva entre rendimientos y tasa de cambio del dólar para la especie TECO2 y negativa para el caso de APBR.

Como puede observarse, los resultados referidos a la influencia de la inflación y el tipo de cambio en el rendimiento de las acciones en el mercado argentino son divergentes y su comparabilidad se dificulta por diversas razones: escasez de estudios, diferencia en las ventanas temporales, discrepancia en factores explicativos incluidos y en la medición de los mismos, entre otros. Por tal motivo, no es posible identificar un efecto unívoco al respecto, destacándose la necesidad (ya marcada en 2002 por Swoboda) de extender y profundizar los estudios empíricos sobre el tema en el mercado de capitales argentino.

Respecto de las tasas de interés libres de riesgo, se observa un efecto dual. Por un lado, el rendimiento de los bonos del tesoro de EEUU exhibe un coeficiente positivo y altamente significativo (modelo I). Este vínculo positivo es similar a lo encontrado por Raele (2011) para la especie FRAN (BBVA Banco Francés) y por Swoboda (2002) quien emplea una tasa de interés en dólares para aproximar la relación. Por su parte, si se ajusta el rendimiento de los T-bonds adicionando el riesgo país de Argentina (EMBI) el coeficiente se torna negativo y significativo (modelos II a V), en consonancia con hallazgos de Swoboda (2002). Esto, en primer lugar, demuestra la importancia de ajustar las tasas libres de riesgo de acuerdo al riesgo soberano de cada país. En segunda instancia, puede ser un indicio del gran impacto negativo del riesgo institucional sobre los rendimientos de títulos privados que cotizan en el país, revertiendo la dirección del efecto (de positivo a negativo).

En referencia a los rendimientos del mercado medidos en moneda local (\$), a través de las variaciones sobre la cotización del índice Merval o el Burcap, el efecto es positivo, tal como se predice en los modelos de equilibrio dentro de las finanzas y de forma coincidente con los hallazgos de Raele 
(2011) y Swoboda (2002). En moneda extranjera (US\$), los rendimientos de mercado medidos a través de los mismos índices presentan un efecto negativo sobre el rendimiento de los activos, lo que podría indicar que posibilidades de inversión alternativas desvían los fondos hacia inversiones internacionales. No se seleccionan modelos que incluyan simultáneamente el premio del riesgo de mercado debido a que estas variables están altamente correlacionadas con las de rendimiento de mercado en US\$ para cualquiera de los índices, por lo que podría presentarse un problema de multicolinealidad.

En cuanto a los efectos temporales capturados a través de las dummies para los años 2008, 2011 y 2013 , los resultados son robustos y significativos en los 5 modelos seleccionados. El signo de las variables dicotómicas para el 2008 y 2011 es negativo, en concordancia con lo esperado, debido al efecto de la crisis financiera internacional y la intervención sobre el mercado cambiario. En el año 2013, la variable presenta signo positivo, posiblemente capturando el salto en las series inflacionarias y/o de tipo de cambio. Esto reafirma los resultados del test de estacionariedad de Zivot-Andrews, según el cual las series son estacionarias con quiebres (ya sea en el intercepto, la tendencia o ambos). En particular, en el caso de la inflación y el tipo de cambio, se presenta un quiebre en la observación 102, correspondiente al mes 6 del año 2013. Una observación de los datos en torno a este momento permite esbozar una hipótesis: en términos relativos y absolutos la variación positiva de la inflación o el tipo de cambio supera la variación negativa del rendimiento, por lo que la dummy temporal asume un signo positivo, contrarrestando el efecto de la variable macro sobre el rendimiento del activo.

En relación al cambio de intercepto por efectos sectoriales, puede afirmarse que en términos generales no se presentan resultados significativos. Sin embargo, el sector de energía y distribución presenta un rendimiento menor que el industrial (resultados significativos en modelos I y II) y también menores que el sector bancario (resultados significativos en el modelo IV). Tampoco se presentan resultados significativos para efectos fijos por empresa, por ello no se incluyen estas variables dicotómicas en los modelos seleccionados.

Debido a la existencia de cointegración, posiblemente los resultados indiquen relaciones de largo plazo, aunque en contextos de mercados emergentes, de poco tamaño y alta concentración, economías inflacionarias y de baja estabilidad institucional, las variables explicativas pueden presentar numerosos cambios estructurales. Por ende, el empleo del vocablo "largo plazo" debe interpretarse en el marco de lo antedicho, teniendo presente las particularidades de la economía durante el período bajo estudio en este país.

\section{Conclusiones.}

Este trabajo investiga empíricamente los factores macroeconómicos vinculados con el rendimiento requerido de un conjunto de acciones en el mercado de capitales argentinos, a través de un modelo tipo fijación de precios por arbitraje, haciendo especial hincapié en la inflación. Para el desarrollo del trabajo, se propone un tratamiento de la temática mediante una investigación descriptiva cuantitativa (correlacional) y en la última etapa, explicativa. Para ello se trabaja con un enfoque de base teóricoempírica. A efectos de plantear el modelo formalmente se realiza un abordaje teórico del tema, con base principalmente en artículos científicos vinculados a la relación entre la tasa de rendimiento de activos y la inflación. El análisis empírico está centrado en el estudio del rendimiento de las acciones de 19 empresas que cotizan en el mercado de capitales argentino en el lapso 2005-2014 y las vinculaciones con variables de interés, en especial macroeconómicas, con algunas pruebas de control con variables microeconómicas. En los párrafos a continuación se sintetizan y discuten los principales hallazgos. 
Con respecto a los indicadores macroeconómicos de mercado, los resultados son robustos en relación al efecto sobre el retorno accionario nominal de las siguientes variables explicativas: rendimiento libre de riesgo (internacional: positivo; con riesgo país: negativo), rendimiento de mercado (en moneda local: positivo; en moneda extranjera: negativo); efectos temporales de períodos "bisagra" (año 2008 y 2011: negativo; año 2013: positivo) y efectos sectoriales (energía y distribución: negativo). Según estos hallazgos, los retornos nominales de las 19 acciones analizadas que integran el Merval 25 se asocian de forma positiva con la tasa libre de riesgo aproximada con la serie T-bonds (es decir, sin incorporar explícitamente el efecto del contexto argentino) y con el rendimiento del índice de mercado medido en pesos. Sin embargo, al incluir el riesgo país usando $T$ bonds $+E M B I$ o al medir el rendimiento de mercado en dólares, se revierte el signo de la relación entre estas variables y los rendimientos nominales de las 19 empresas estudiadas, pasando a ser negativo. Este cambio en la dirección de la asociación entre los retornos accionarios estudiados y las variables explicativas "tasa libre de riesgo" y "rendimiento de mercado" en sus diferentes versiones (internacional/con riesgo país; pesos/dólares, respectivamente) exhibe la importancia de incluir en los modelos de valoración de acciones aspectos específicos del contexto examinado, como lo son el factor de riesgo local y el comportamiento de los índices de mercado en moneda local y extranjera. Asimismo, la evidencia obtenida resalta la relevancia de utilizar modelos APT en un contexto emergente y cambiante como el argentino, cuyas particularidades tornan dificultosa la utilización de métodos de fijación de precios con más restricciones y menos parámetros (por ejemplo, CAPM o DCAPM). Esto coincide con lo expresado por Raele (2011) quien encuentra para el mercado de capitales argentino una mayor eficiencia del modelo APT respecto al resto.

En relación a las variables monetarias, los estudios internacionales o realizados en mercados desarrollados (principalmente EEUU) son numerosos y proporcionan evidencia empírica relativamente homogénea sobre la relación entre rendimientos accionarios e inflación, en general contradictoria al efecto de Fisher. Sin embargo, no sucede lo mismo con las investigaciones efectuadas en contextos emergentes, donde los trabajos son escasos y sus hallazgos divergentes. Específicamente, los resultados del presente documento no son determinantes en referencia al impacto de las variables inflación y tipo de cambio sobre el rendimiento de las acciones. En ese sentido, los modelos estimados con índices de variación de precios oficiales del INDEC (que exhiben una inflación contenida y de un nivel no tan elevado como las mediciones alternativas) parecen indicar que las acciones representan una cobertura contra la pérdida de poder adquisitivo de la moneda (vínculo positivo). En cambio, cuando las regresiones se efectúan con IPC de fuentes no oficiales (que implican una mayor depreciación monetaria) se registra un deterioro en el rendimiento de los activos ante mayores niveles de inflación. En este último caso, la relación entre retornos accionarios nominales e inflación se invierte pasando a ser negativa y concuerda con la evidencia empírica mayoritaria opuesta al efecto de Fisher.

En función de lo expresado en el párrafo precedente, se destaca la necesidad de extender y profundizar los estudios sobre valoración de activos de mayor riesgo en mercados poco desarrollados y altos niveles de inflación como el argentino para determinar el "verdadero" efecto de las variables monetarias sobre los rendimientos de las firmas. Esto resulta una tarea compleja, considerando tres fenómenos: i) el estudio de un mercado, que no solo se caracteriza como emergente, sino que presenta una inestabilidad política e institucional alta, con riesgo de expropiaciones, defaults por parte del Estado, turbulencias sociales o políticas y barreras al libre flujo de capitales; ii) el período bajo estudio, con atributos particulares en cuanto al mercado cambiario y la intervención del INDEC para la medición de la inflación; y iii) la escasez de información sobre variables explicativas clave no incluidas en las regresiones por ausencia de estacionariedad e imposibilidad de trabajar con sus diferencias (por ejemplo, inversión en activos).

Para finalizar con la síntesis de resultados empíricos, es importante destacar que el indicador macroeconómico de actividad aproximado con la variable PIB, si bien no se incluye en las estimaciones multivariadas por cuestiones de periodicidad de las series de datos disponibles presenta, 
en los análisis bivariados de correlación, una relación positiva y alta con los rendimientos de las acciones.

Dentro de las contribuciones del presente trabajo, más allá de los hallazgos específicos comentados ut supra, una de las fortalezas está representada en el empleo de múltiples proxies para una misma variable de interés. En particular, la inflación fue testeada a partir de cinco medidas alternativas, el tipo de cambio con tres series, el rendimiento libre de riesgo a partir de cuatro títulos, el rendimiento de mercado mediante dos índices en dos monedas y la prima de riesgo a través de dos proxies (Tabla A.1 del Anexo). Asimismo, el hecho de trabajar con datos de panel representa una diferencia a la mayoría de estudios que abordan la temática, siendo la variable dependiente el rendimiento individual de las acciones, y no el rendimiento de un índice de mercado de manera agregada.

A modo de limitación puede mencionarse la carencia de series mensuales sobre algunas variables relevantes, así como la falta de actualización de la base con datos de los últimos 5 períodos. De todas formas, el grupo de investigación pretende continuar estudiando en profundidad el fenómeno a fin de encontrar una explicación robusta para las variables inflación y tipo de cambio. Para ello, se plantea sortear las limitaciones anteriormente mencionadas, intentar completar la base de datos (inicialmente diseñada con 25 empresas) y extender su amplitud temporal incorporando el rango 2015-2019. Asimismo, como futuras líneas de investigación se propone considerar otras pruebas complementarias y especificaciones diferentes sobre los modelos regresados, testeando por ejemplo el efecto de la inflación rezagada en varios períodos, regresiones individuales por empresa y nuevos indicadores para las variables explicativas.

\section{Referencias}

Ang, A., Brière, M., \& Signori, O. (2012). Inflation and individual equities. http://basepub.dauphine.fr/handle/123456789/7847

Balduzzi, P. (1995). Stock returns, inflation, and the 'proxy hypothesis': A new look at the data. Economics Letters, 48(1), 47-53.

Bodie, Z. (1976). Common Stocks as a Hedge against Inflation. The Journal of Finance, 31(2), 45983.

Branch, B. (1974). Common stock performance and inflation: An international comparison. Journal of Business, 47(1), 48-52.

Burmeister, E., \& Wall, K.D. (1986). The Arbitrage Pricing Theory and Macroeconomic Factor Measures. Journal of Financial Review, 21, 1-20.

Cagan, P. (1974). Common Stock Values and Inflation - The Historical Record of Many Countries. Supplement to NBER Report Thirteen, 1-10. http://www.nber.org/chapters/c4221.pdf.

Chen, N.F., \& Ingersoll, J.E. (1983). Exact Pricing in Linear Factor Models with Finitely Many Assets: A Note. The Journal of Finance, 38, 985-988.

Choudhry, T. (2001). Inflation and rates of return on stocks: evidence from high inflation countries. Journal of International Financial Markets, Institutions and Money, 11(1), 75-96. 
Ciner, C. (2015). Are equities good inflation hedges? A frequency domain perspective. Review of Financial Economics, 24, 12-17.

Dapena, J.P. (2013). La evolución del índice Merval (precio de acciones) y la inflación. Centro de Economía Aplicada. Universidad del CEMA, Departamento de Finanzas.

Fama, E.F. (1981). Stock returns, real activity, inflation, and money. The American Economic Review, $71(4), 545-565$.

Fama, E.F., \& French, K.R. (1993). Common risk factors in the returns on stocks and bonds. Journal of Financial Economics, 33(1), 3-56.

Fama, E.F., \& MacBeth, J.D. (1974). Tests of the multiperiod two-parameter model. Journal of Financial Economics, 1(1), 43-66.

Fama, E.F., \& Schwert, G.W. (1977). Asset returns and inflation. Journal of Financial Economics, 5(2), 115-146.

Firth, M. (1979). The Relationship between Stock Market Returns and Rates of Inflation. The Journal of Finance, 34(3), 743-757.

Fornero, R. (2003). Análisis Financiero en Condiciones de Inflación. Documento de trabajo. Universidad Nacional de Cuyo.

Geske, R., \& Roll, R. (1983). The fiscal and monetary linkage between stock returns and inflation. The Journal of Finance, 38(1), 1-33.

Greene, W.H. (2003). Econometric analysis. $5^{\text {th }}$ edition. New Jersey, USA: Pearson Education.

Guerra, A.L. (2012). Rendimiento de las acciones en contextos inflacionarios: análisis empírico del mercado accionario argentino. http://www.capacitacion.bcr.com.ar/Documentos/Edicion esBCR/17Rendimiento\%20de\%20las\%20acciones\%20en\%20contextos\%20inflacionarios.pdf

Gujarati, D.N. (2004). Basic econometrics. New York, USA: McGraw-Hill.

Gujarati, D.N., \& Porter, D.C. (2011). Econometria Básica-5. México: McGraw Hill.

Gultekin, N.B. (1983). Stock Market Returns and Inflation: Evidence from Other Countries. The Journal of Finance, 38(1), 49-75.

Hagmann, M., \& Lenz, C. (2004). Real asset returns and components of inflation: a structural VAR analysis. International Center for Financial Asset Management and Engineering. http://www.swissfinanceinstitute.ch/print/rp118.pdf

Hudson, R., \& Gregoriou, A. (2010). Calculating and Comparing Security Returns is harder than you think: A comparison between logarithmic and simples returns. SSRN https://papers.ssrn.com/sol3/papers.cfm?abstract_id $=1549328$

Jaffe, J.F., \& Mandelker, G. (1976). The «Fisher Effect» for Risky Assets: An Empirical Investigation. The Journal of Finance, 31(2), 447-458.

Johnson, G.L., Reilly, F.K., \& Smith, R. E. (1971). Individual common stocks as inflation hedges. Journal of Financial and Quantitative Analysis, 6(3), 1015-1024. 
Kaul, G. (1987). Stock returns and inflation. Journal of Financial Economics, 18, 253-276.

Kennedy, P. (2008). A guide to econometrics. Malden, USA: John Wiley \& Sons.

Lanche-Bode, M. (2015). Análisis de las consecuencias de la inflación en el mercado de capitales argentino [Tesis de Maestría en finanzas, Universidad Nacional de Rosario]. https://rephip.unr.edu.ar/xmlui/handle/2133/11329

López, W. (2016). Inflación y rendimientos accionarios en Argentina [Tesis de Maestría en finanzas, Universidad de San Andrés]. http://repositorio.udesa.edu.ar/jspui/handle/10908/12067

Marshall, D.A. (1992). Inflation and asset returns in a monetary economy. The Journal of Finance, 47(4), 1315-1342.

Montero, R. (2013). Variables no estacionarias y cointegración. Documentos de Trabajo en Economía Aplicada, Universidad de Granada, España. http://www.ugr.es/ montero/matematicas/cointegracion

Nelson, C.R. (1976). Inflation and rates of return on common stocks. The Journal of Finance, 31(2), 471-483.

Nichols, D. (1976). Discussion. The Journal of Finance, 31, 483-487.

Oudet, B.A. (1973). The Variation of the Return on Stocks in Periods of Inflation. Journal of Financial and Quantitative Analysis, 8(2), 247-258.

Raele, N.A. (2011). Optimización de portfolios de inversión en países emergentes: el caso argentino. [Tesis de grado en Ingeniería Industrial, Instituto Tecnológico Buenos Aires]. https://ri.itba.edu.ar/handle/123456789/410

Reilly, F.K., Johnson, G.L., \& Smith, R. E. (1970). Inflation, inflation hedges, and common stocks. Financial Analysts Journal, 26(1), 104-110.

Roll, R., \& Ross, S. (1980). An empirical investigation of the Arbitrage Pricing Theory. The Journal of Finance, 35(5), 1073-1103.

Ross, S. (1976). The arbitrage theory of capital asset pricing. Journal of Economic Theory, 13, 341360.

Solnik, B. (1983). The Relation between Stock Prices and Inflationary Expectations: The International Evidence. The Journal of Finance, 38(1), 35-60.

Swoboda, C.J. (2002). Teoría del Arbitraje de precios: una investigación empírica para la Argentina. Documentos de trabajo $\mathrm{N}^{\circ}$ 15. Universidad Nacional de Córdoba, Facultad de Ciencias Económicas, Departamento de Economía y Finanzas. http://www.biblioeco.unsa.edu.ar/pmb/opac_css/index.php?lvl=bulletin_display\&id=4699 
Wooldridge, J.M. (2002). Econometric analysis of cross section and panel data. MIT press. Cambridge.

Wooldridge, J.M. (2015). Introductory econometrics: A modern approach. 6th edition. Boston, USA; Cengage learning.

\section{Anexos}

Tabla A.1. Diferentes indicadores para cada variable explicativa testeada.

\begin{tabular}{|l|l|}
\hline Variables de inflación & Rendimiento de mercado \\
\hline Inflación_INDEC base04 & r-Merv\$ \\
\hline IPCCong & r-MervUS\$ \\
\hline IPCBevaq & r-MervU\$S \\
\hline IPCpricest & r-BurcU\$S \\
\hline CVSprivform & Premio por riesgo de mercado \\
\hline Tipo de cambio & PRM(merv) \\
\hline TCNof & PRM(burc) \\
\hline TCNpa & Nivel de actividad \\
\hline TCNcliqui & PIB_cte 04 \\
\hline Tasa de rendimiento libre de riesgo & PIB_corr \\
\hline Rf tbonds & Controles \\
\hline Rf_tbond+EMBI & Sector (efecto fijo) \\
\hline Rf3_Disc & Empresa (efecto fijo) \\
\hline Rf4_Boden & Año (efecto temporal) \\
\hline
\end{tabular}

Fuente: Elaboración propia. 
Tabla A.2. Análisis de estacionariedad de las series temporales.

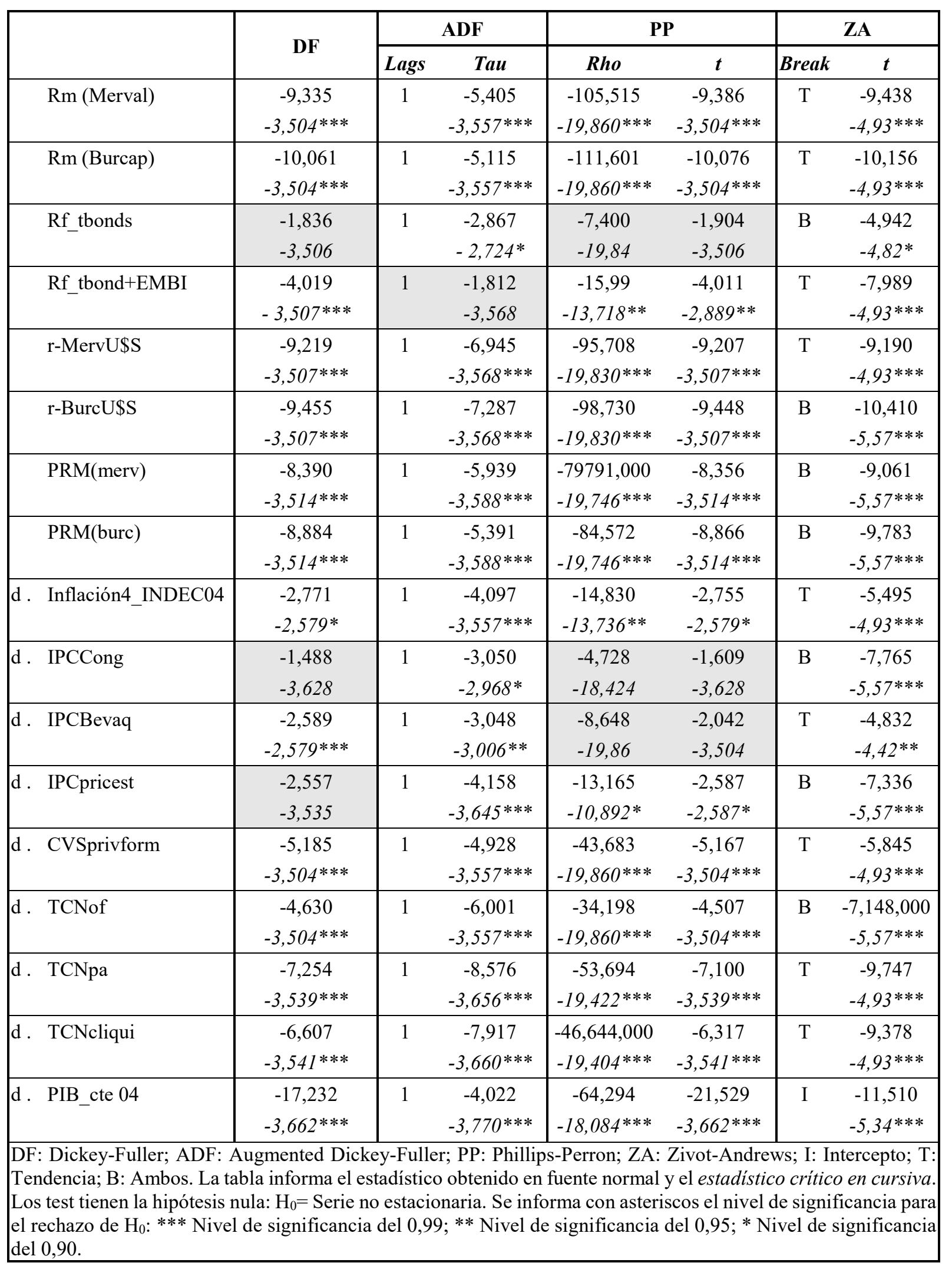

Fuente: Elaboración propia. 
Ilustración A.1. Serie temporal de los residuos del modelo I.

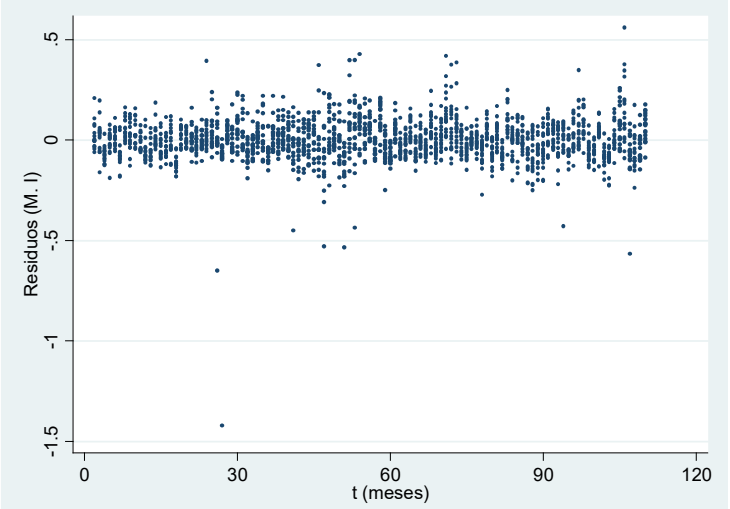

Fuente: Elaboración propia con base en software econométrico.

Ilustración A.4. Serie temporal de los residuos del modelo IV.

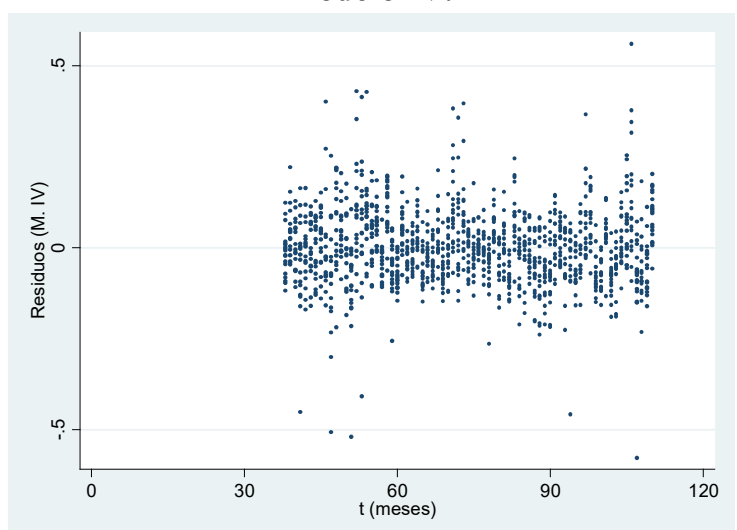

Fuente: Elaboración propia con base en software econométrico.
Ilustración A.2. Serie temporal de los residuos del modelo II.

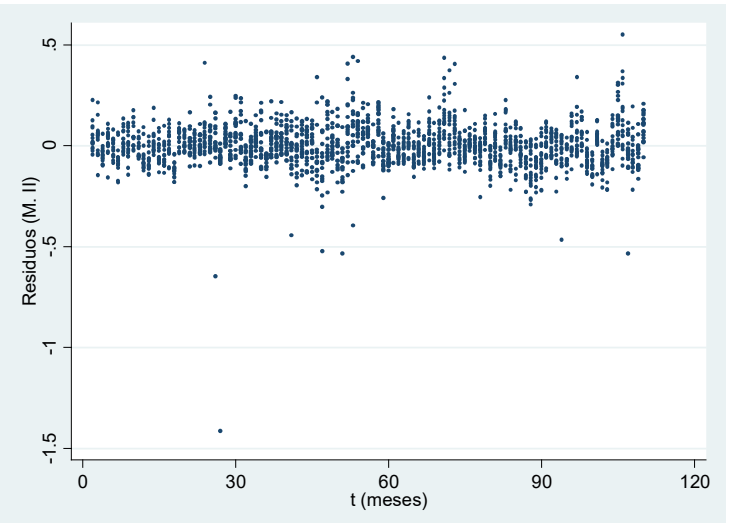

Fuente: Elaboración propia con base en software econométrico.

Ilustración A.5. Serie temporal de los residuos del modelo V.

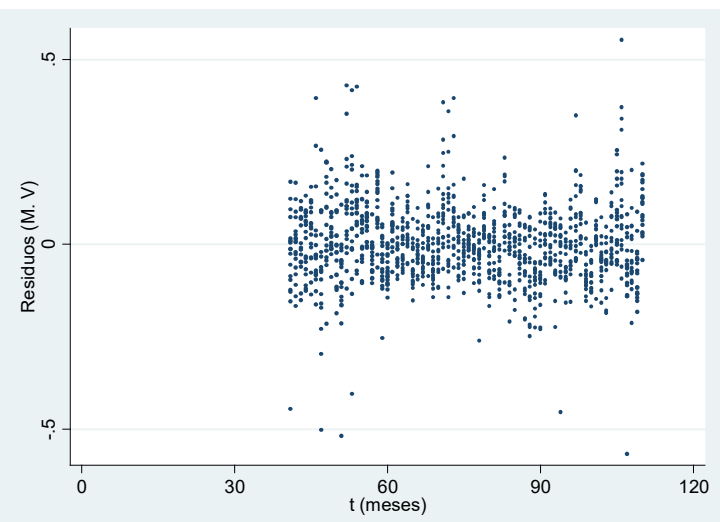

Fuente: Elaboración propia

con base en software econométrico.
Ilustración A.3. Serie temporal de los residuos del modelo III.

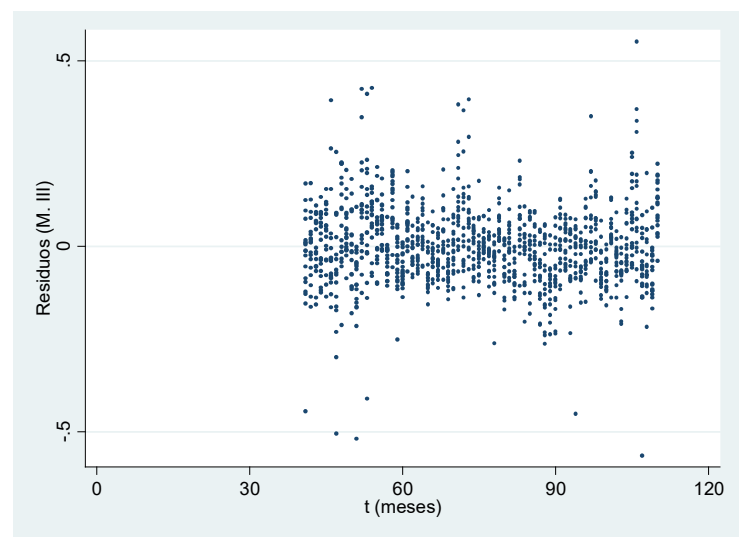

Fuente: Elaboración propia con base en software econométrico. 